\title{
AN INVESTIGATION ON THE PERFORMANCE LEVEL OF RC SHEAR WALL-FRAME SYSTEMS UTILIZING NONLINEAR ANALYSIS
}

\section{Fadwa Issa,}

PHD student, Department of Structural Engineering, Faculty of Civil Engineering, Damascus University

e-mail: fadwa.issa650@gmial.com

\author{
Mohamad Nazih Alyagchi Eilouch ${ }^{1}$ and Abbas Tasnimi ${ }^{2}$ \\ ${ }^{1}$ Professor, Department of Structural Engineering, Faculty of Civil \\ Engineering, Damascus University, Yarmouk University \\ e-mailnazihayagchi@gmail.com \\ ${ }^{2}$ Professor, Department of Structural Engineering, International Institute \\ of Earthquake Engineering and Seismology (IIEES), Iran \\ e-mail tasnimi@gmail.com
}

(Received July 26, 2011 Accepted September 5, 2011)

Prediction of performance level of the reinforced concrete Shear wallFrame structural systems are not only dependent on the method of nonlinear analysis, but are also related to some effective parameters used in each method. This article studies the effect of some important parameters such as plastic rotational capacity of the structural elements, capacity spectrum, drift and the overall damage of the building, from two viewpoints, one of which is the performance level of structural elements and also the performance level of the overall structure neglecting the nonstructural elements. In order to achieve the expected results, 92 nonlinear analyses including 64 nonlinear static analyses and 28 nonlinear dynamic analyses have been carried out on three dimensional buildings comprising 8, 12, 16 and 20 stories. These buildings were analyzed and designed according Syrian concrete code of practice (2004).

All of the nonlinear analyses were carried out on the bases of the above mentioned parameters as a main criteria utilizing IDARC-ver6 program. The obtained results show that the performance evaluation based on overall displacement of the whole structure and the failure of an element, is a restriction of capacity curve which has provoked some doubts.

KEY WORDS: Shear wall-Frame Systems, Nonlinear analysis. 


\title{
دراسة حول تقييم مستوى الأداء لأنظمة إطار- جار قص خرسانية باستخدام تحليل لاخطي
}

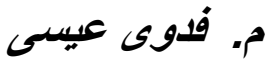 \\ طالبة دكتوراه في قسم المندسة الإنشائية

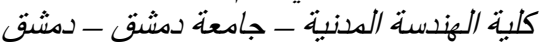 \\ e-mail:fadwa.issa650@gmail.com
}

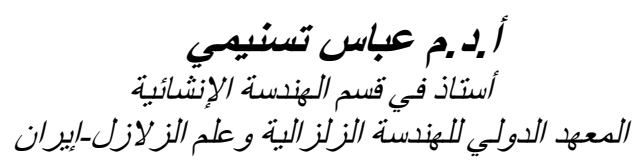

e-mail: nazihayagchi@gmail.com

\author{
أ ـد.م محمد نزيه اليضشي إبليوش \\ أستاذ في قسم الهندسة الإنشائية

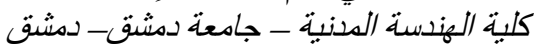 \\ كلبة الهندسة المدنية - جامعة البيرموك - درعانة \\ e-mail: tasnimi@gmail.com
}

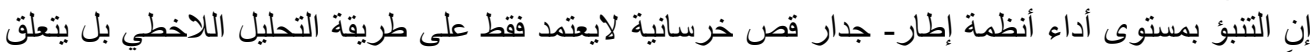

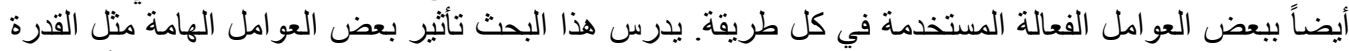

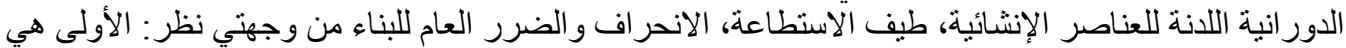

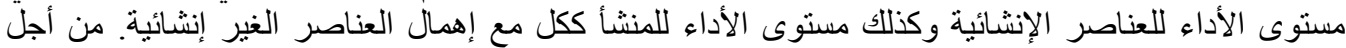
تحقيق النتائج المتوقعة فقد تم تنفيذ 92 تحليل لاخطي يتضئنس

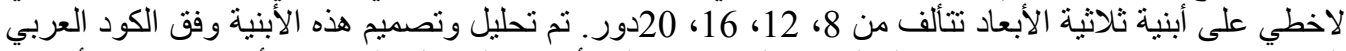

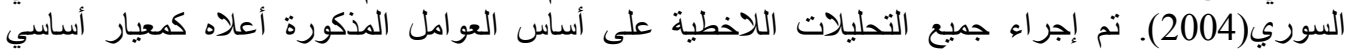

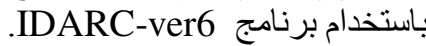
تبين النتائج الني حصلنا عليها أن تقييم الأداء اعتماداً على التشكل العام للمنشأ ككل و على انهيار عنصر يقيد

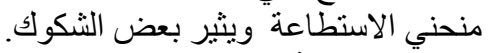
كلمات مفتاحية: أنظمة إطار -جدار قص، التحليلة التكات اللاخطية.

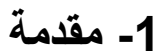

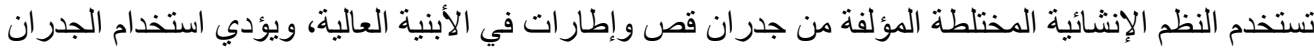

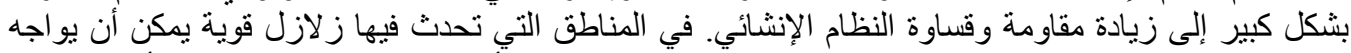

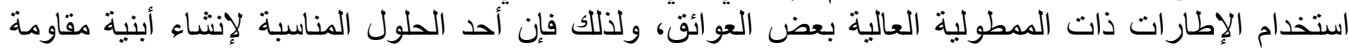

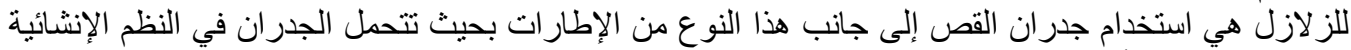

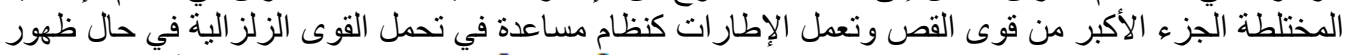

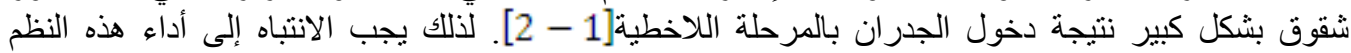

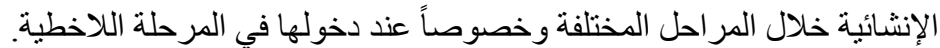

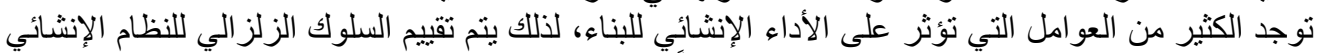

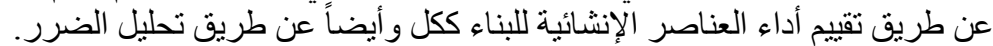

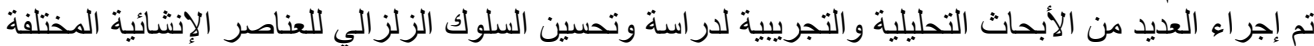

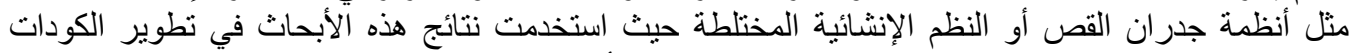

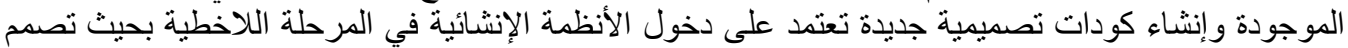

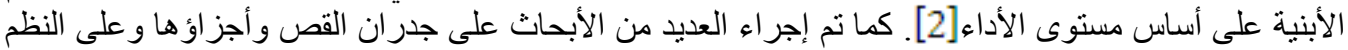

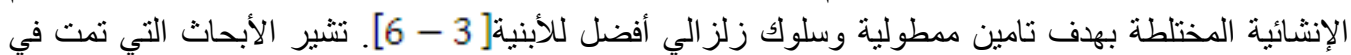

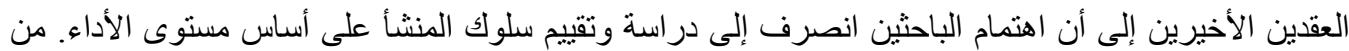

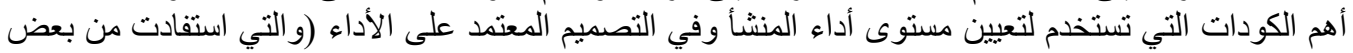




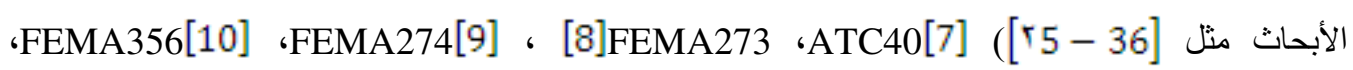

.FEMA307[12] ،FEMA306[11]

تم في هذا البحث دراسة مستوى أداء النظم الإنشائية المختلطة مع الأخذ بعين الاعتبار مجموعة من العوامل

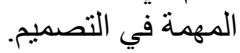

\section{2- مراحل تعيين مستويات الأداء}

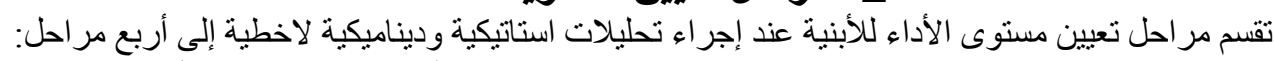

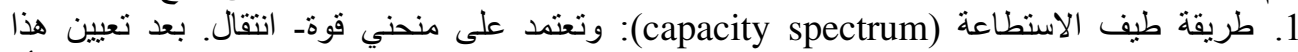

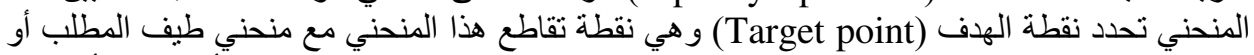
طيف ألزلزال (demand spectrum). وبعد تعيين نقطة الهدف ينم تعيين مستوى أداء المنشأ (يوجد شر ح مفصل لهذه الطريقة في (ATC40).

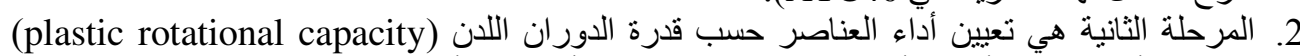
باستخدام أحد كودات تأهيل الأبنية ومن ثم بتحدد مستوى أداء الداء المنثأ.

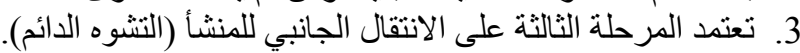

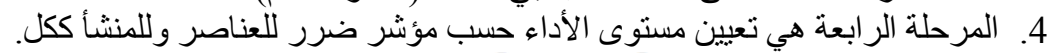

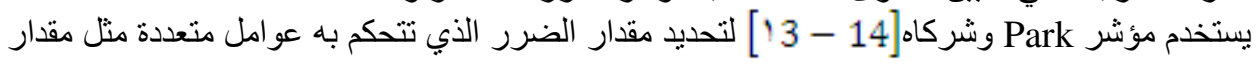

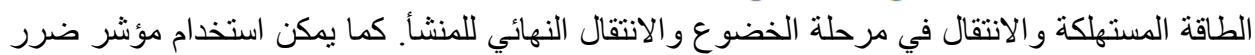
Ghobarah المنشأ ككل اعنماداً على هذه المؤشرات التئ.

\section{3- مؤشر الضرر}

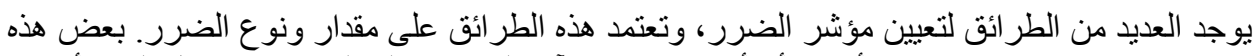

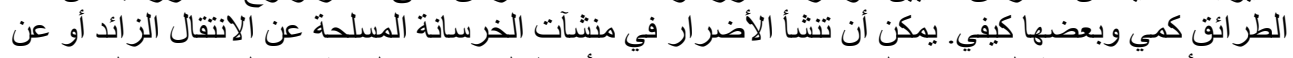

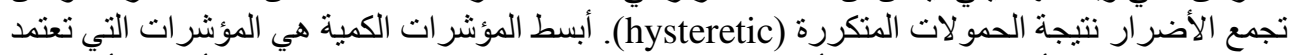

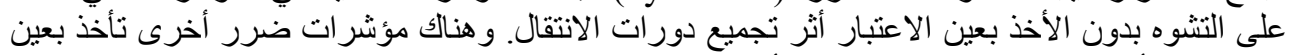

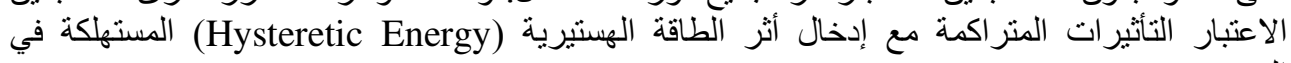

\section{1-3 - 2 - 1ائشرات المحلية}

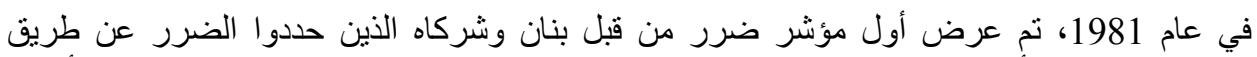

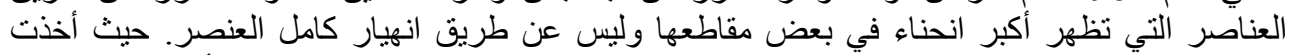

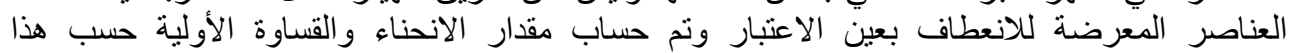

الشرط[16].

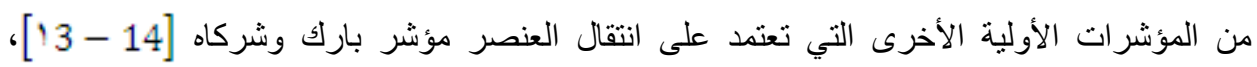

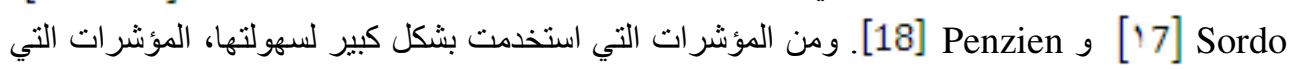

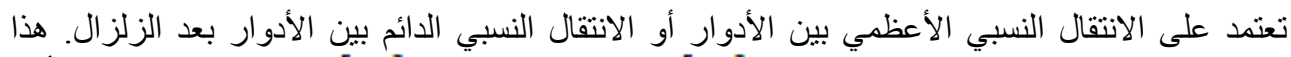

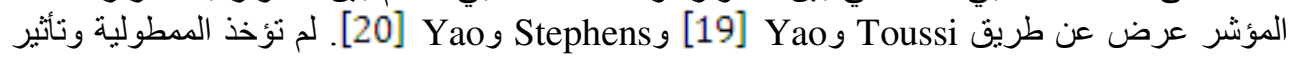

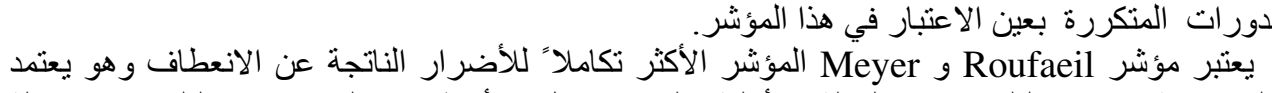
على نسبة زيادة التلان من الحالة الأولية إلى الانتقال الأعظمي على زئلى زيادة التلان في حالة

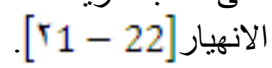

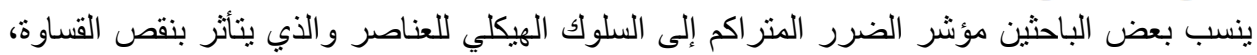

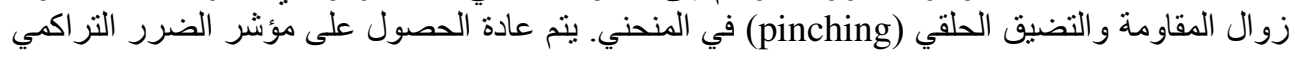


بالاستفادة من إحدى صيغ التعب التي يكون فيها مؤشر الضرر على شكل تابع لتغيير الشكل اللان المتر اكم

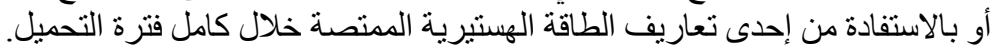

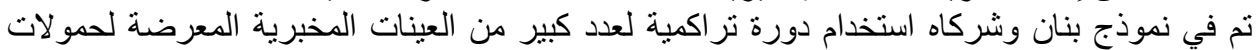

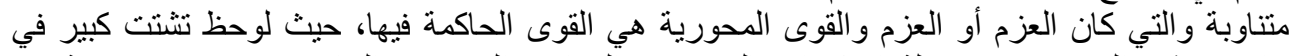

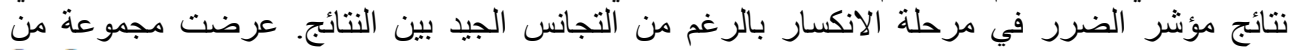

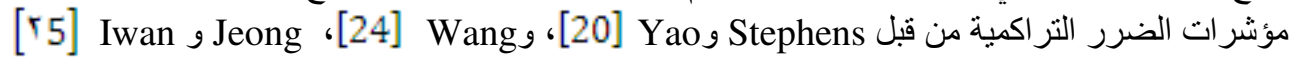

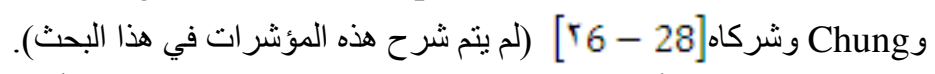

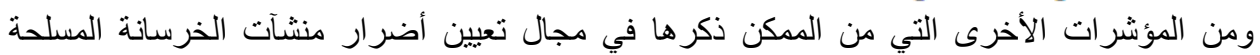

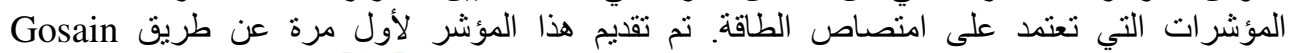

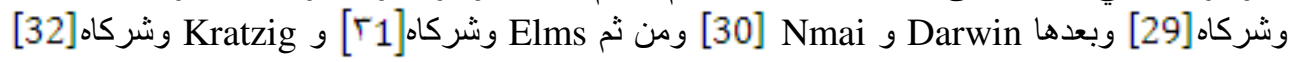

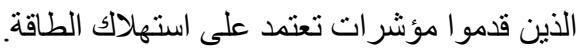
إن أكثر مؤشرات الضرات أنرات التراكية شهرة والذي يستعمل في الكثير من المجالات هو مؤشر

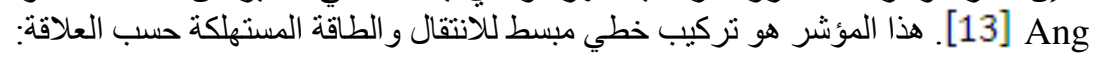

$$
\mathrm{DI}=\frac{\delta_{\mathrm{mm}}}{\delta_{\mathrm{ul}}}+\frac{\beta}{\delta_{\mathrm{ul}} \times \mathrm{f}_{\mathrm{y}}} \int \mathrm{dE}
$$

8u

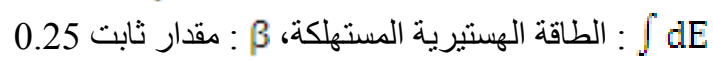
الجزء الأول من العلاقة معيار مبسط عن الانتقال شبه الاستاتيكي، أما الضرر التراكمي (الجزء المتعلق

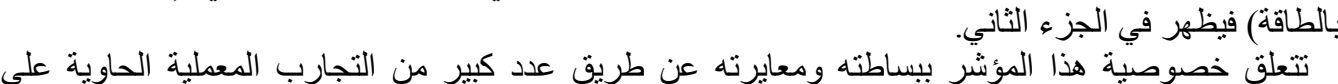

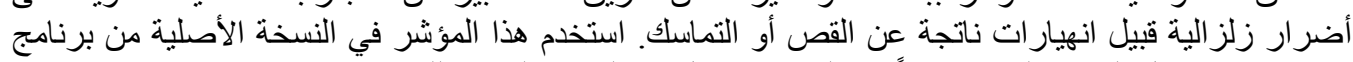
IDARC

$$
\mathrm{DI}=\frac{\theta_{\mathrm{m}}-\theta_{\mathrm{r}}}{\theta_{\mathrm{u}}-\theta_{\mathrm{r}}}+\frac{\dot{\beta}}{\mathrm{M}_{\mathrm{y}}, \theta_{\mathrm{u}}} \int \mathrm{Eh}
$$

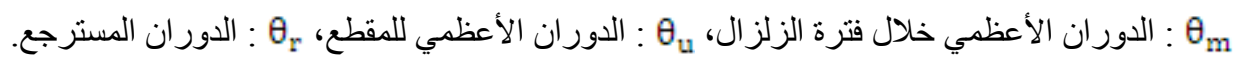

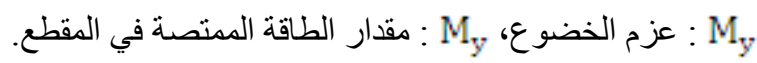

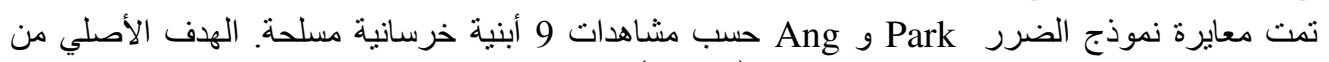

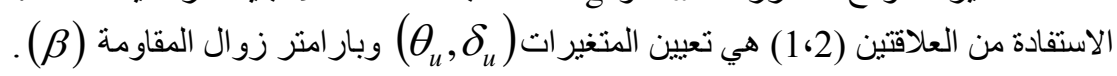

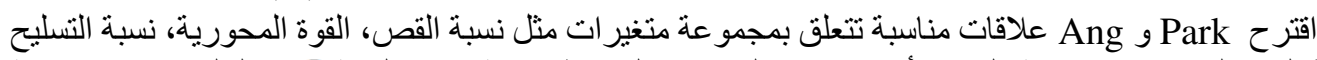

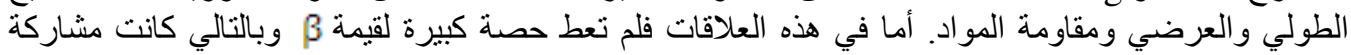

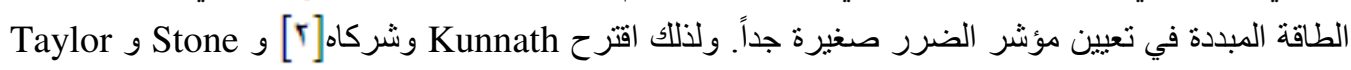

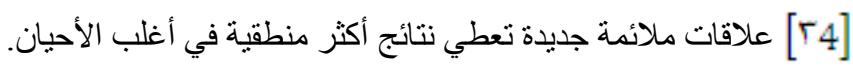

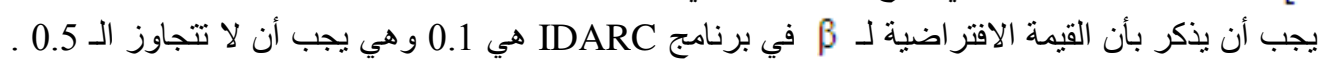
القيمة المختارة يجب أن تعبر عن زو ال المقاومة في النموذج الهينتيري.

2-3 مؤشرات الضرر الكلية (العامة)

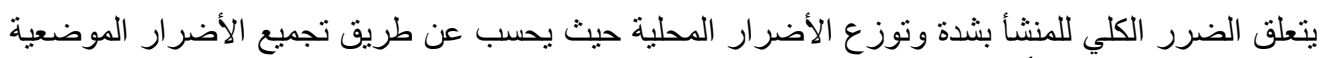

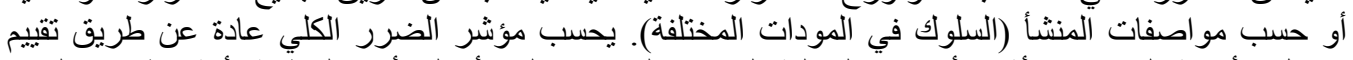

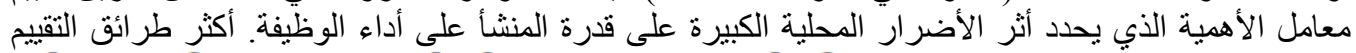

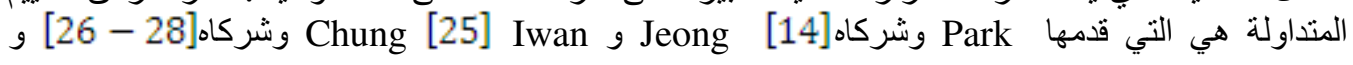


Kunnath أهمية) حسب امتصاص الطاقة. وِبنفس الطريقة يحسب مؤشر الضرر لكامل المنشأ من مؤشر ات الضرات الضرر للأدوار.

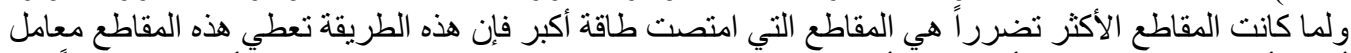

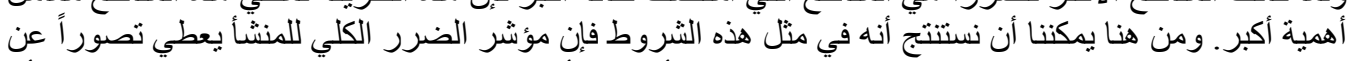

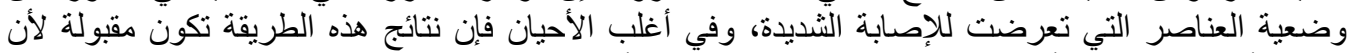

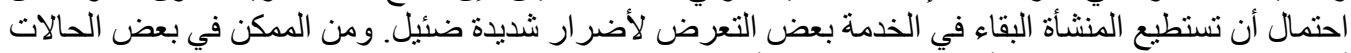

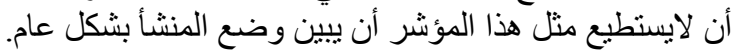

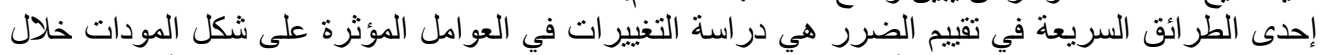

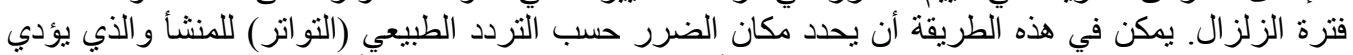

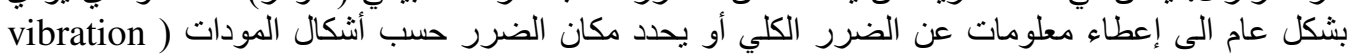

(modes

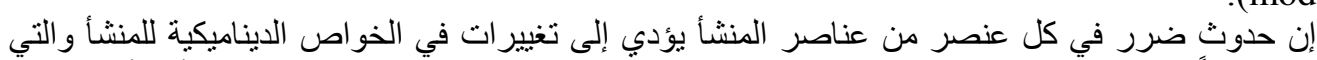

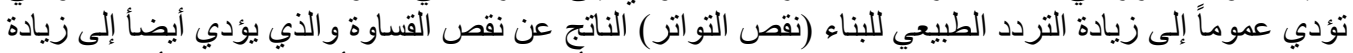

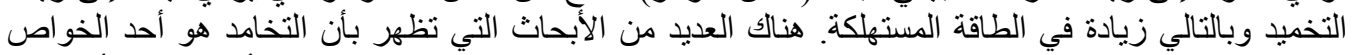

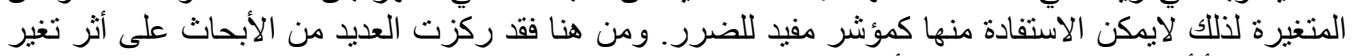

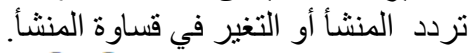

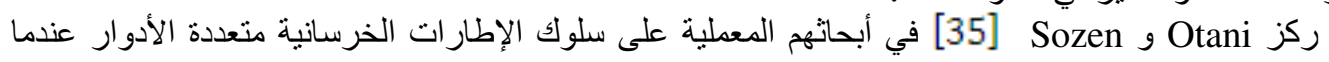

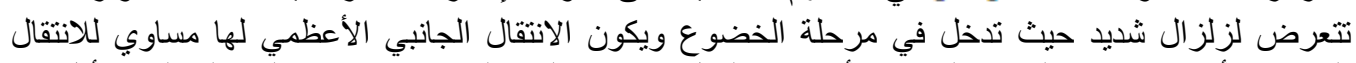

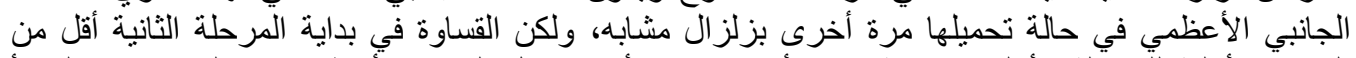

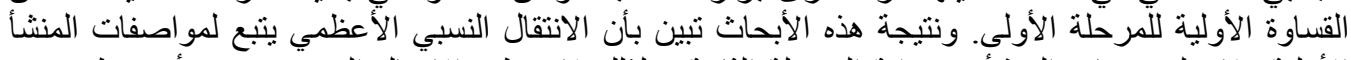

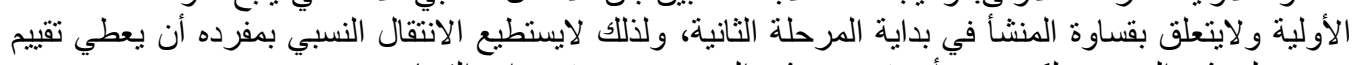

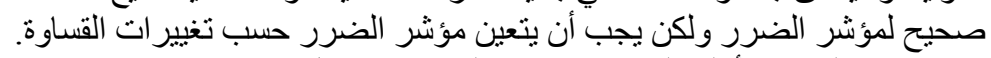

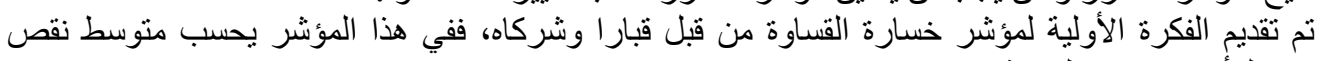

$$
\mathrm{DI}=\left(1-\frac{\mathrm{K}_{\text {final }}}{\mathrm{K}_{\text {initial }}}\right)
$$
القساوة للأدوار حسب العلاقة الاولة

K Kinitial ، مؤشر نقص القساوة (يتراوح من 0 عند عدم وجود ضرر إلى 1 في حالة الضرر الثديد) : DI

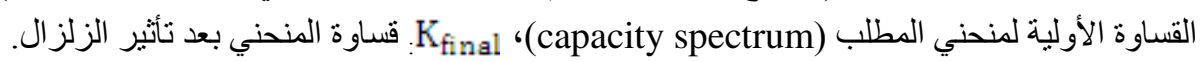

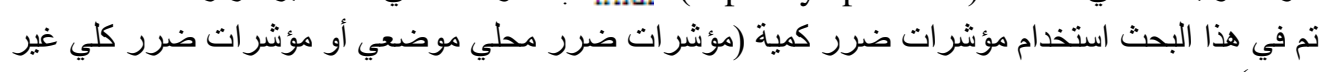

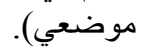

\section{4- مواصفات الابنية المدروسة}

تم في هذا البحث تحليل أربعة أبنية من الخرسانة المسلحة مصممة وفق الكود العربي السوري2004، وهئ وهي

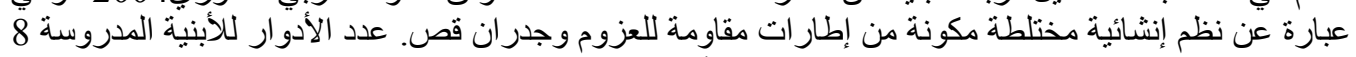

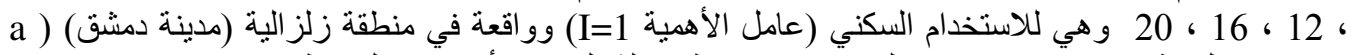

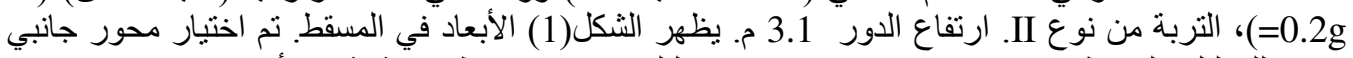
واحد للتحليل اللاخطي (بالاتجاه Y) (1). تم إجراء تحليل استاتيكي خطي تثلاثي الأبعاد باستخدام برنامج 


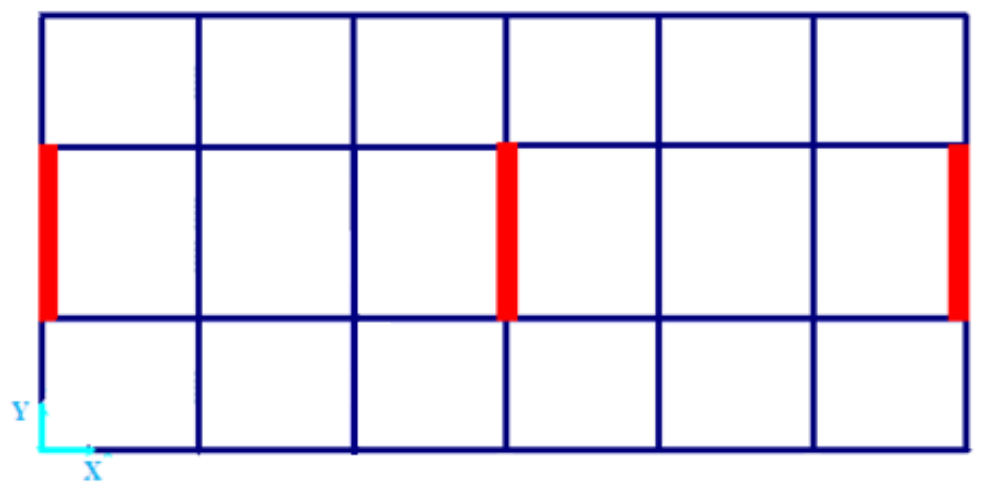

$$
\text { الثكل(1): المسقط الأفقي للنماذج الددروسة. }
$$

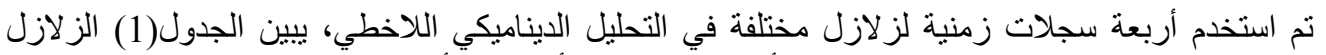

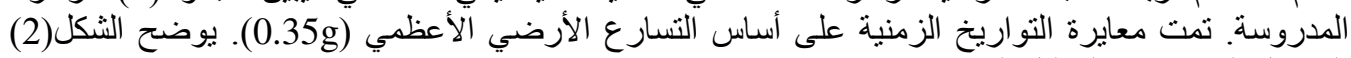

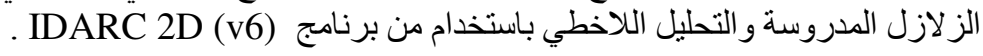
الجدول(1): مواصفات الزلازل المستخدمة

\begin{tabular}{|c|c|c|c|}
\hline نوع التربة & $\begin{array}{c}\text { التسارع الأرضي الأعظمي } \\
\text { PGA } \\
\text { (g) }\end{array}$ & سنة الحدوث & اسم الزلزال \\
\hline II & 0.357 & 1989 & لوما بريتا (Loma Prieta) \\
\hline II & 0.25 & 1986 & بالم اسبرينك (N-Palm Spring) \\
\hline II & 0.256 & 1994 & نوتريدج (Northridge) \\
\hline II & 0.366 & 1971 & سان فرناندو (Sanfernando) \\
\hline
\end{tabular}

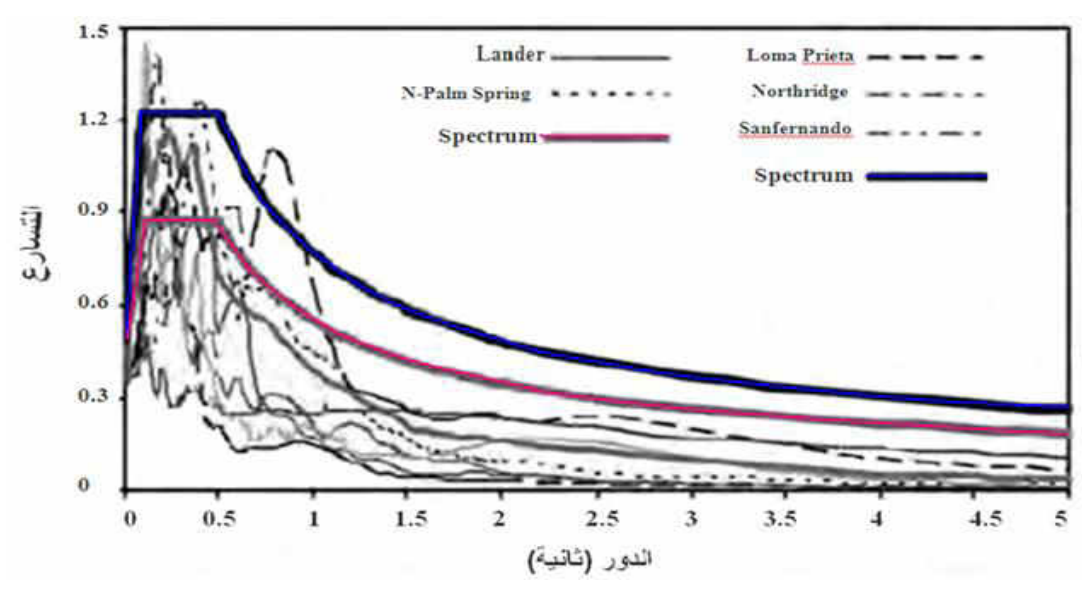

الثكل(2): مخطط التسار ع وطيف الزلازل المستخدمة في تحليل التاريخ الزمني 


\section{5- تعبين مستوى الاداء}

1-5 المرحلة الاولى- طيف الاستطاعة(capacity spectrum)

تم الحصول على منحني القوة - الانتقال أو منحني استطاعة المنشأ عن طريق التحليل الاستاتيكي اللاخطي.

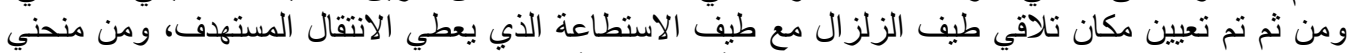

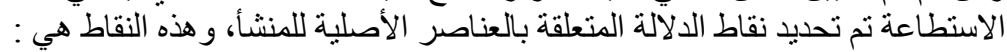

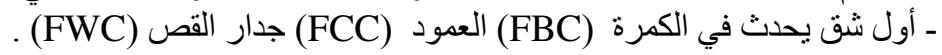
ـ أول تلان يحدث في الكمرة (FBY) العمود (FCY) جدار القص (FWY) .

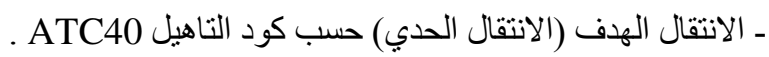

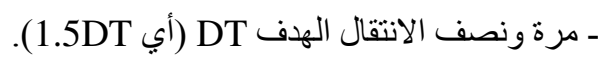

وفق الطريقة A من الكود ATC40 يحسب الانتقال الموافق لمستوى الأداء لكل منشأ ويوضح على منحني

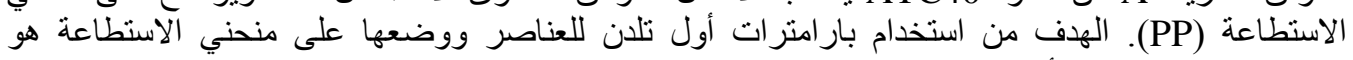

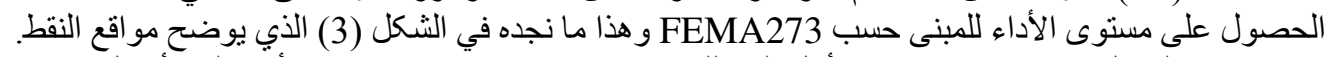

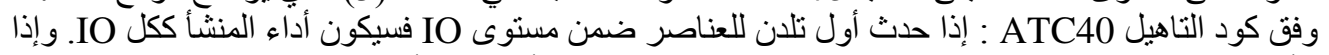

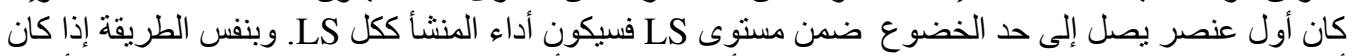

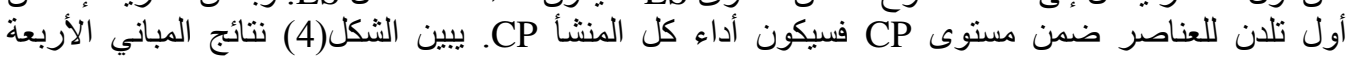
المدروسة (تم تصغير مقياس منحني الاسنطاعة في هذا الثكل لتوضيح المجال اللاخطي للمباني).

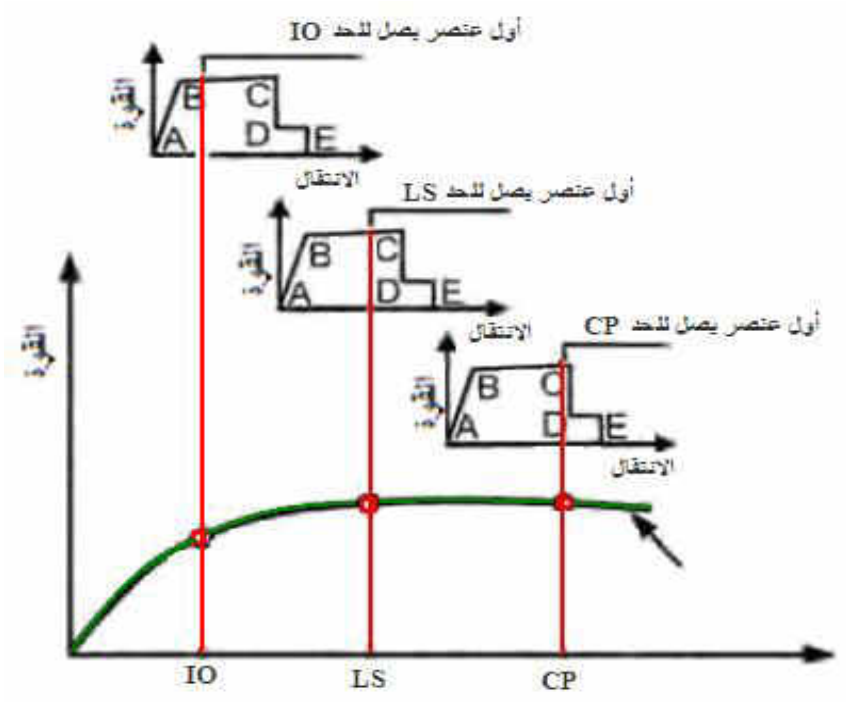

[8] FEMA273 الثكل(3): معيار مستويات الأداء وفق

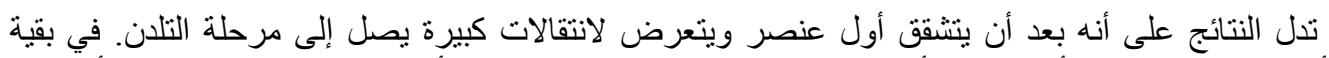

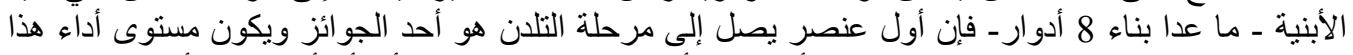

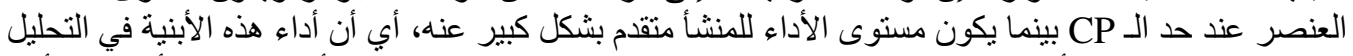

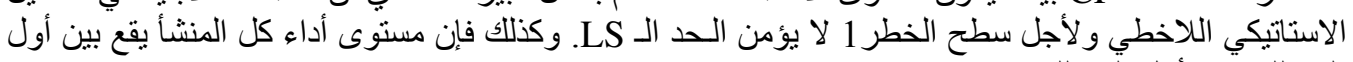

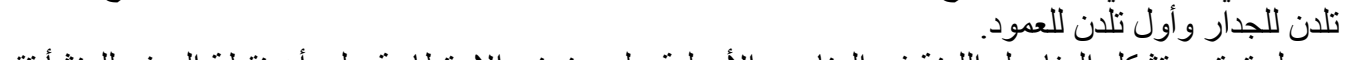
يدل نرتيب تشكل المفاصل اللانة في العناصر الأصلية على منحني الاستطاعة على أن نقطة الهدف للمنشأ تقع

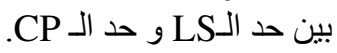




\section{2-5 المرحلة الثانيةــ مقدار دوران العناصر اللانة}

يحدد مقدار الدوران اللان لكل عنصر من عناصر النماذج المختلفة باستخدام تحليلات استاتيكية وديناميكية

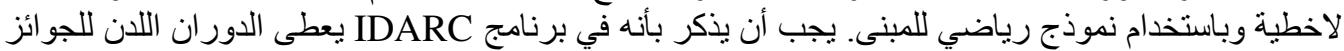

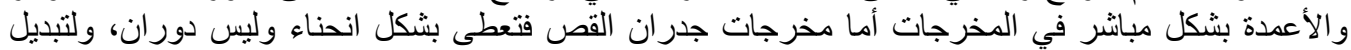

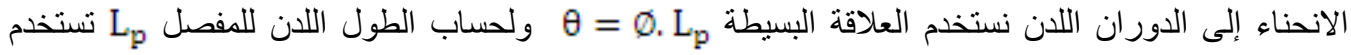

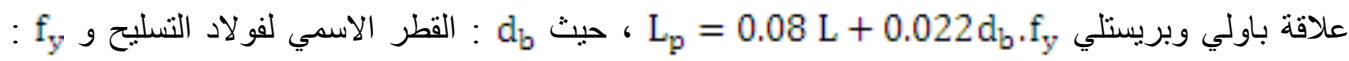

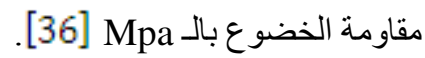

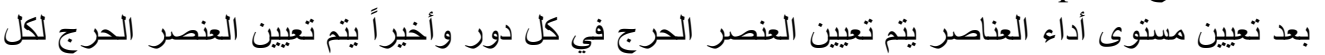

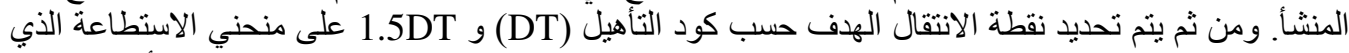
حصلنا عليه من التحليل الاستاتيكي اللاخطي كما يحدد الانتقال الدقابل لحد الانهيار CP المبني على أساس مقدار الدوران اللان لكل عنصر من عناصر المنشاً.

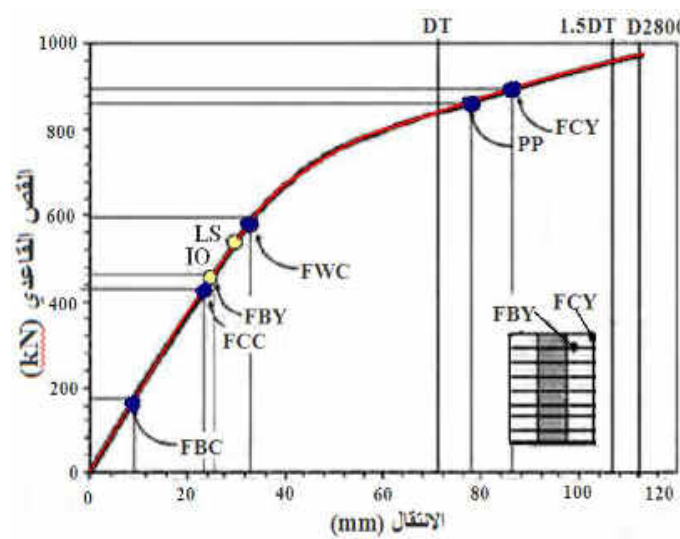

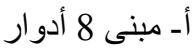

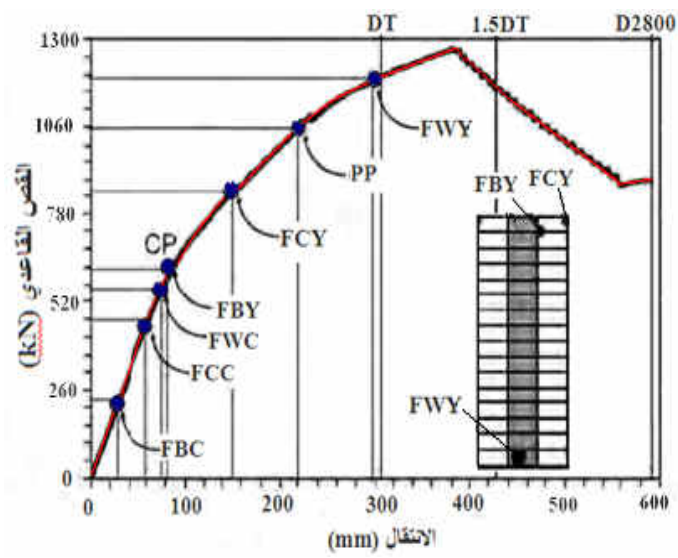

ج- مبنى16 دور

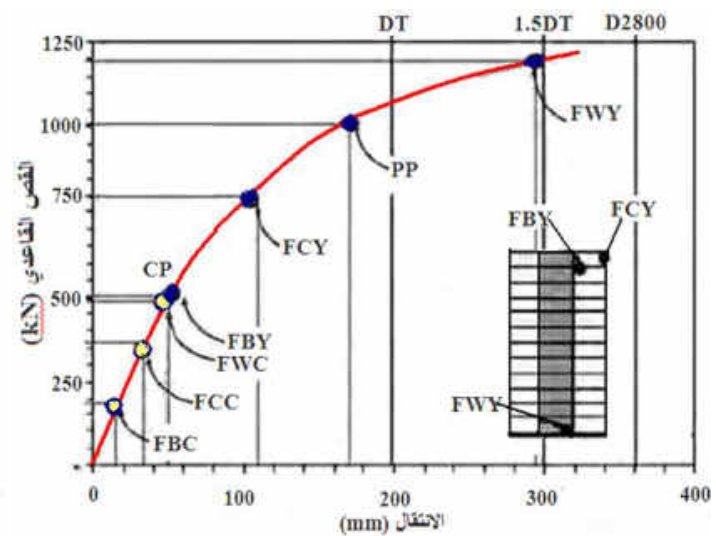

ب- مبنى 12 دور

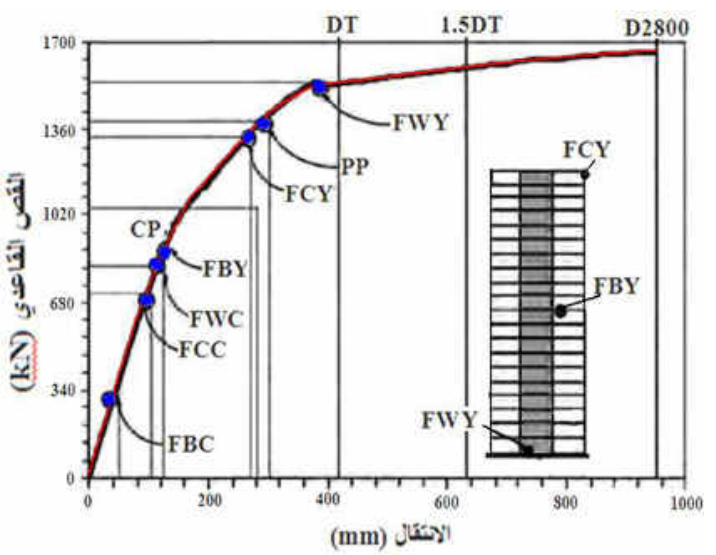

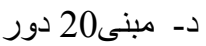

الثكل(4): منحني السعة والسلوك الزلز الي في التحليلات الاستاتيكية اللاخطبة (نموذج توزيع الحمو لات بشكل 
ملاحظة هامة: في التحليلات الديناميكية اللاخطية إذا أدى الزلزال إلى انهيار كل المنشأ ولم يكن من الممكن

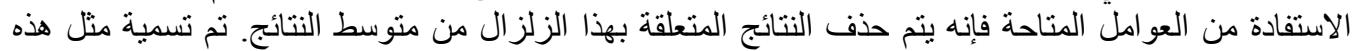

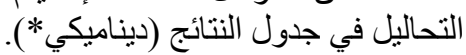

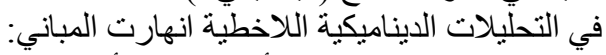

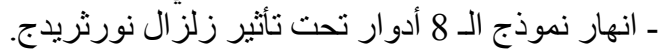
ـ انهار نموذج الـ 12 دور تحت تأثنير زلز ال نورثريدج. ـ انهار نموذج الـ 16 دور تحت تأثثر زلز ال لوما بريتا. ـ في حين لم ينهار نموذج الـ 20 دور تحت تأثير أي من الزلازل المدروسة. تظهر النتائج أن:

• في نموذج الـ 8 أدوار: تمكنت جميع العناصر من تحقيق شروط سطح الخطر 1 (LS) في حد الانتقال الهدف

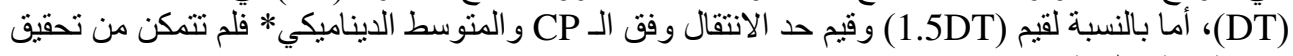

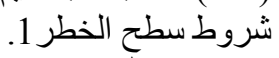
هي نموذج الـ 12 دور : العناصر التي لم تتمكن من تحقيق شروط سطح الخطر (1) هي:

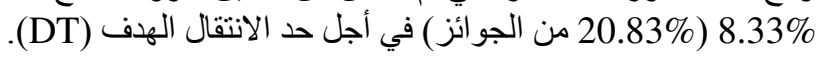

11.67\% (29.17\% من الجو ائز) من أجل مرة ونصف من حد الانتقال الهدف (1.5DT). 41.67\% ( كامل الجوائز و 8.33\% من الأعمدة) في حد الـ \% 15\% (37.5\% من الجوائز) في المتوسط الدينامبكي. و لكن في المتوسط الديناميكي* 60\% من العناصر ( 100\% من الجوائز، 50\%من الأعمدة، 25\% من (وندران القص). • في نموذج الـ 16 دور العناصر التي لم تتمكن من تحقيق شروط سطح الخطر(1) هي:

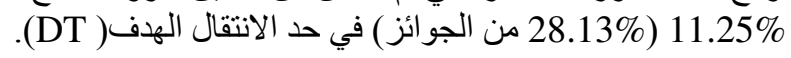

22.5\% (53.13\% من الجوائز و6.25\% من الأعمدة) من أجل مرة ونصف من حد الانتقال الهدف

$62.5 \%$ من العناصر ( 100\% من الجوائز، 5من الأعمدة، 18.75\% من جدران القص) في المتوسط الديناميكي, و لكن في المتوسط الديناميكي* 33.75\% من العناصر ( 81.25\% من من الجوائز، 6.25\% من الأعمدة).

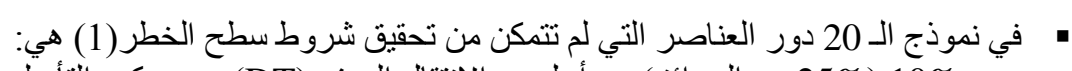

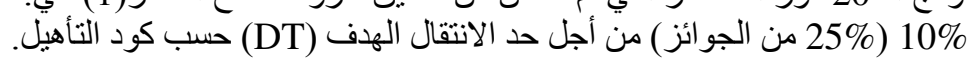
25\% (60\% من الجوائز و 5\% من الأعمدة) من أجل مرة و نصف من حد الانتقال الهذف (1) من

(1.5DT

49\% من العناصر ( 100\% من الجوائز، 45\% من الأعمدة) في المتوسط الديناميكي، ولكن في

$$
\text { و لذلك فإن مستويات الأداء للمباني المدروسة الديناميكي 30\% من العناصر ( 75\% من الجو ائز). }
$$

• للنموذج 8 أدو ار: مستوى الأداء حسب نقطة الانتقال الهدف (DT) و 1.5DT وحسب المتوسط الديناميكي

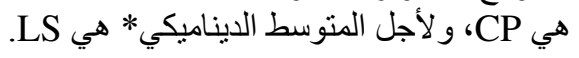




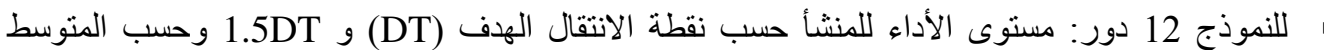

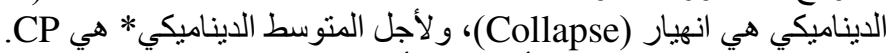

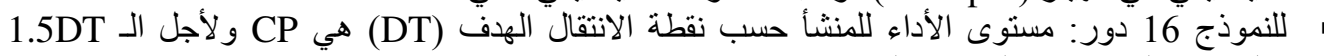
و المتوسط الديناميكي و المتوسط الديناميكي* هي النهيار ( Collapse).

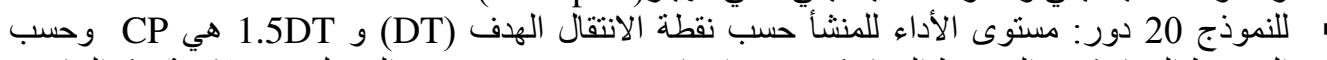

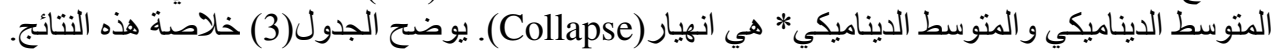

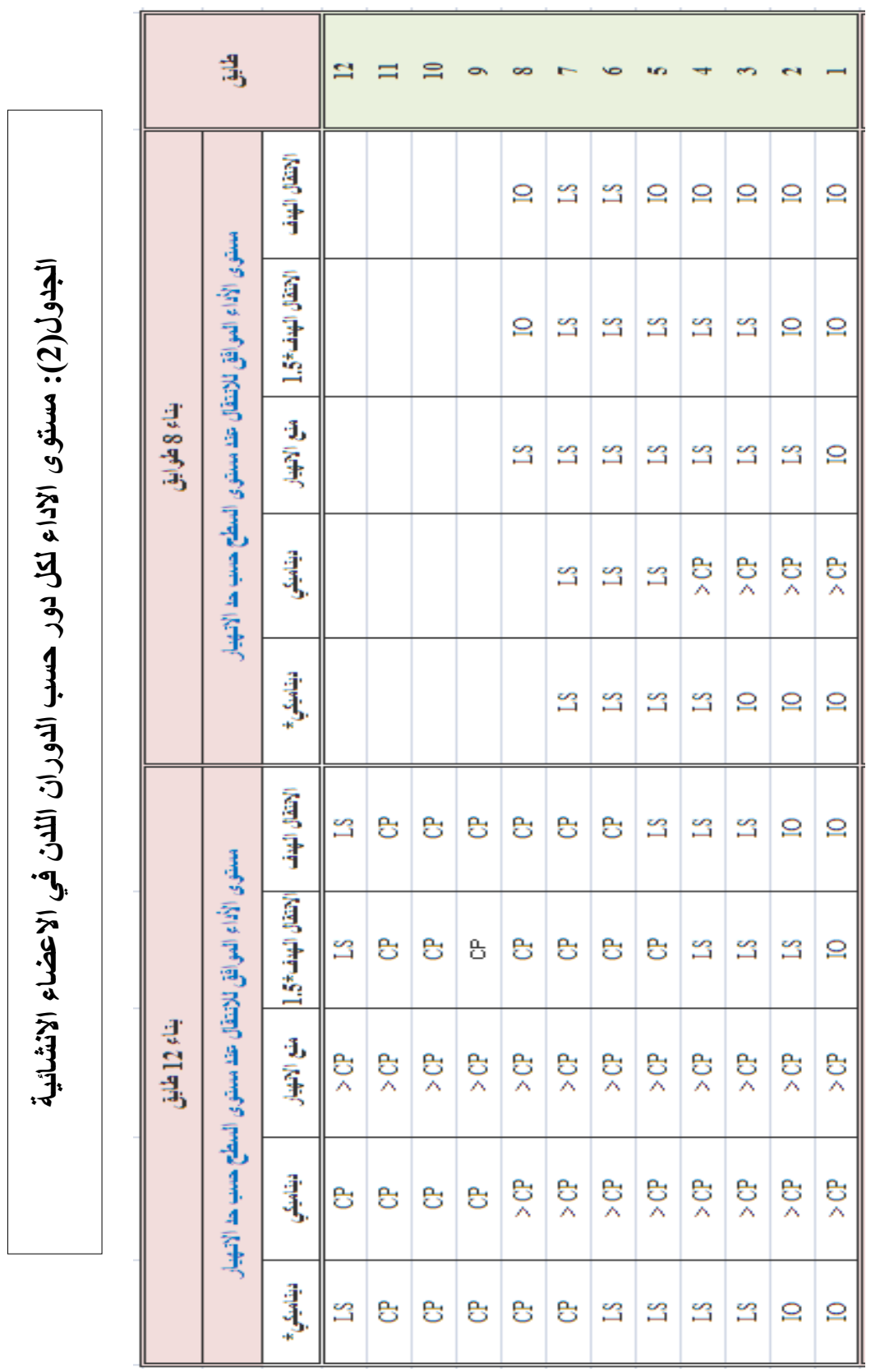




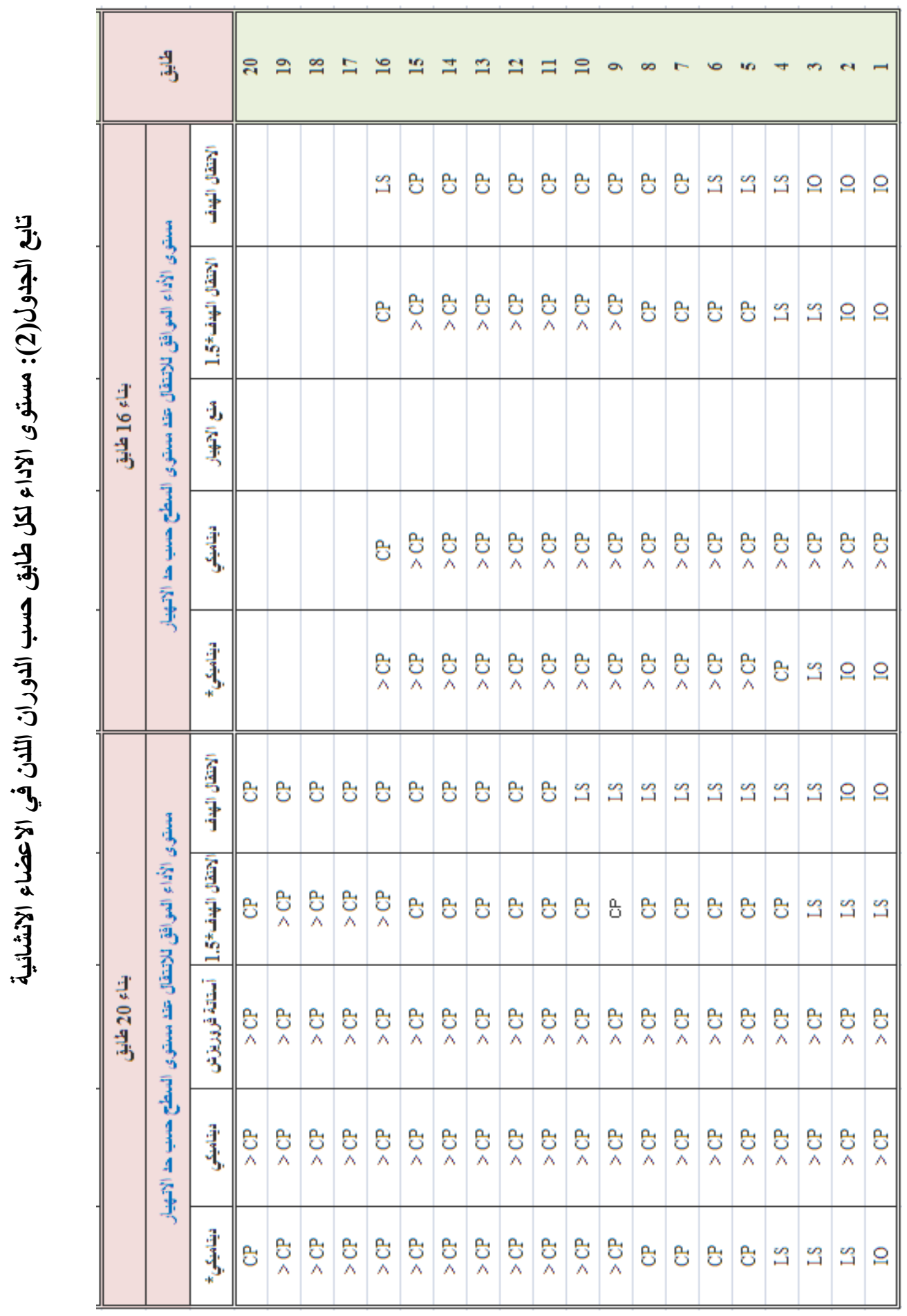




\section{3-5 المرحلة الثالثة ـ مقار الاتثقال النسبي الجانبي}

يتم تحديد مستوى الأداء للمنشأ في هذه المرحلة حسب الانتقال النسبي الجانبي (أو في الو اقع الانتقال الدائم

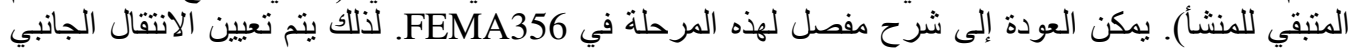

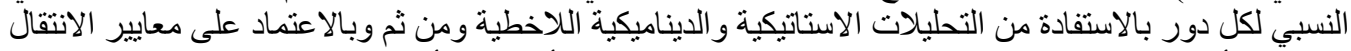

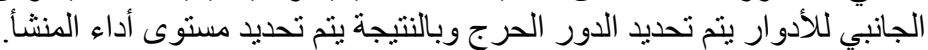

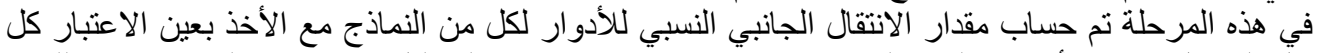

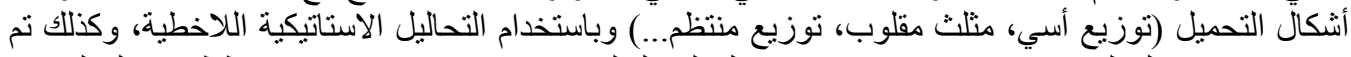

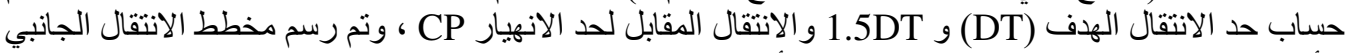
للأدو ار لكل النماذج. تم عرض نتائج التحميل الأسي فقط في الثكل (5) (لهنع التكرار).

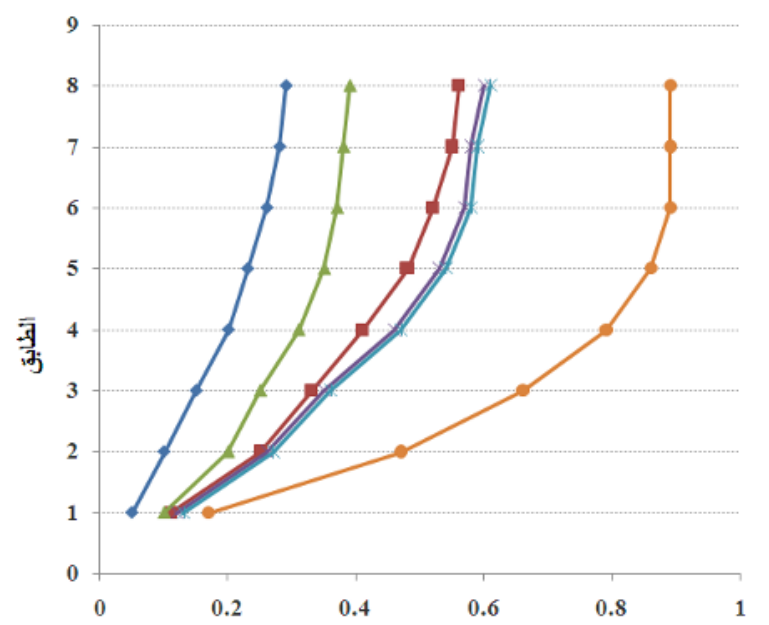

نموذج 8 طوابق

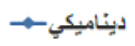

-Loma Prieta

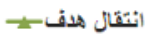

*NP Spring

انتقال الاتهبير -

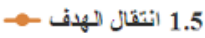

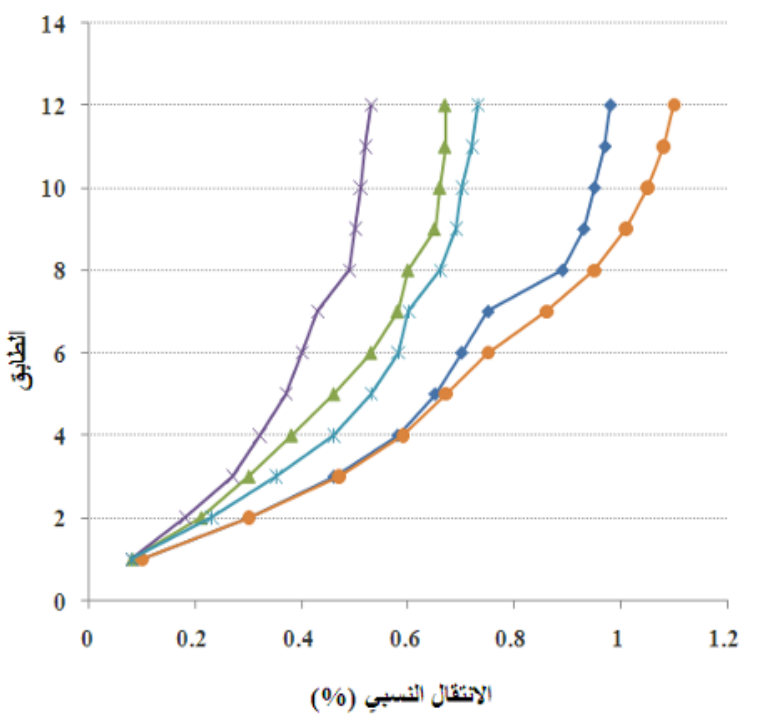

نموذج 12 طو ابق

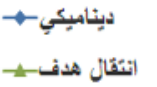

*NPSpring

1.5

*-Sanfernando

الانثقال الثنببي (\%)

الثكل(5): منحني الانتقالات الجانيية النسبية الناتجة عن التحليلات الاستاتيكية اللاخطية (نموذج توزع الحمولات بشكل أسي) والتحليلات الديناميكية اللاخطية 

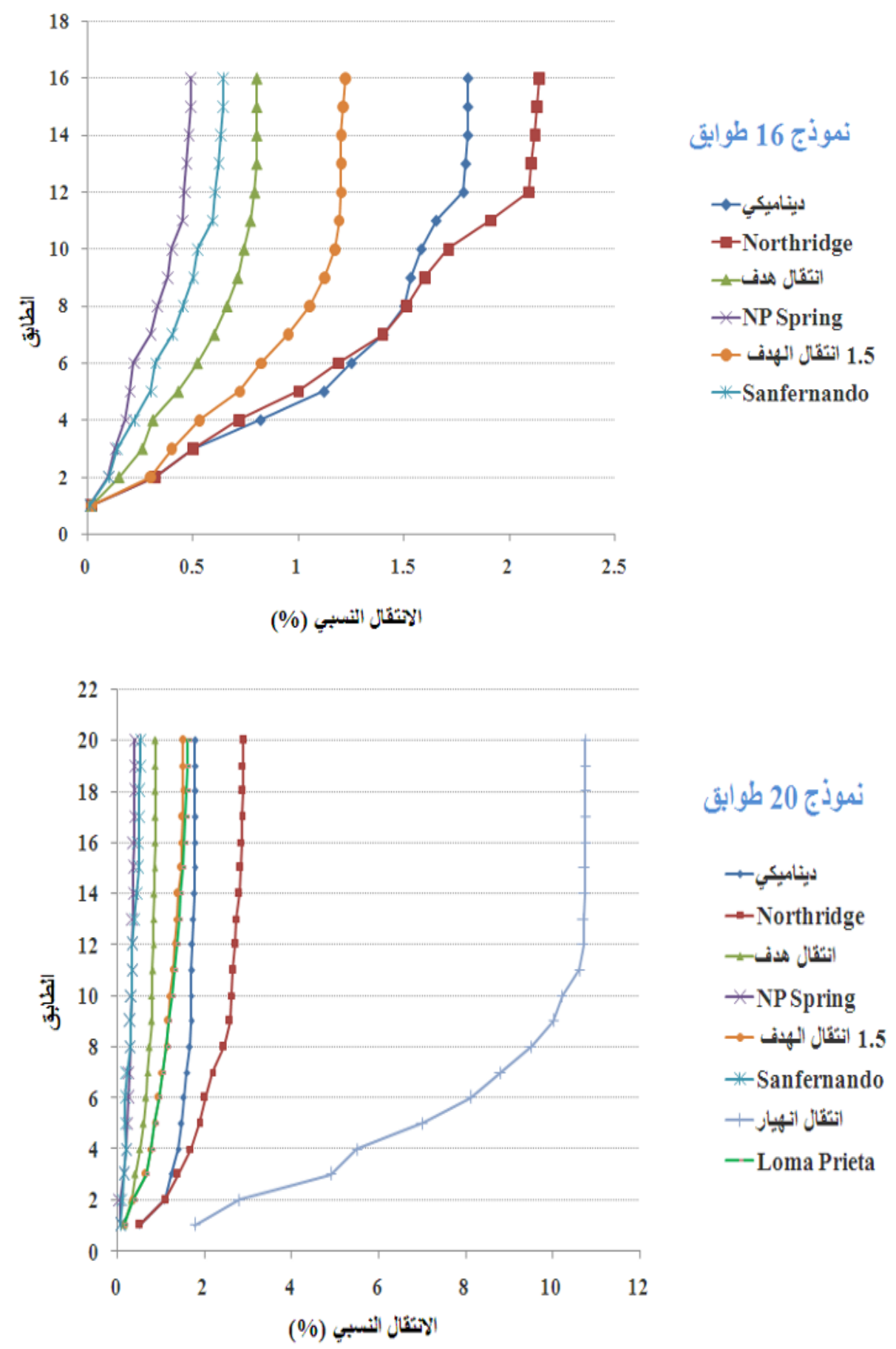

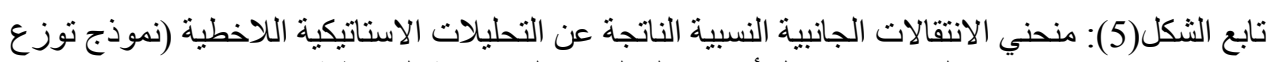
الحمو لات بشكل أسي) و التحليلات النجية الديناميكية الناخطية الانئية 
لدر اسة السلوك الزلز الي للمباني فقد تم فحص بعض النقاط من منحني الاستطاعة مع تغير الانتقالات النسبية وكانت النتيجة:

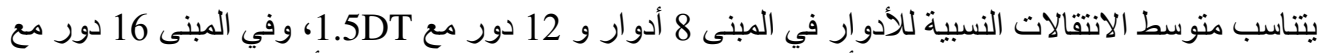

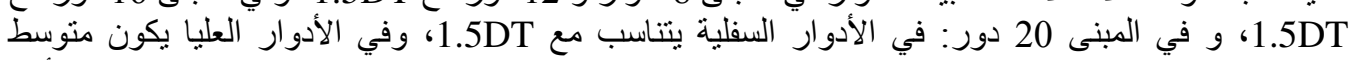
الانتقالات النسبية بين حد الـ 1.5DT و الانتقال المقابل للانهيار. في الجي الجدول (3) و و (4) تم تحديد مستوى الأداء لكل دور بالاعتماد على مقدار الانتقال النسبي الجانبي.

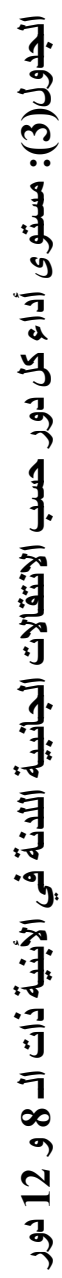

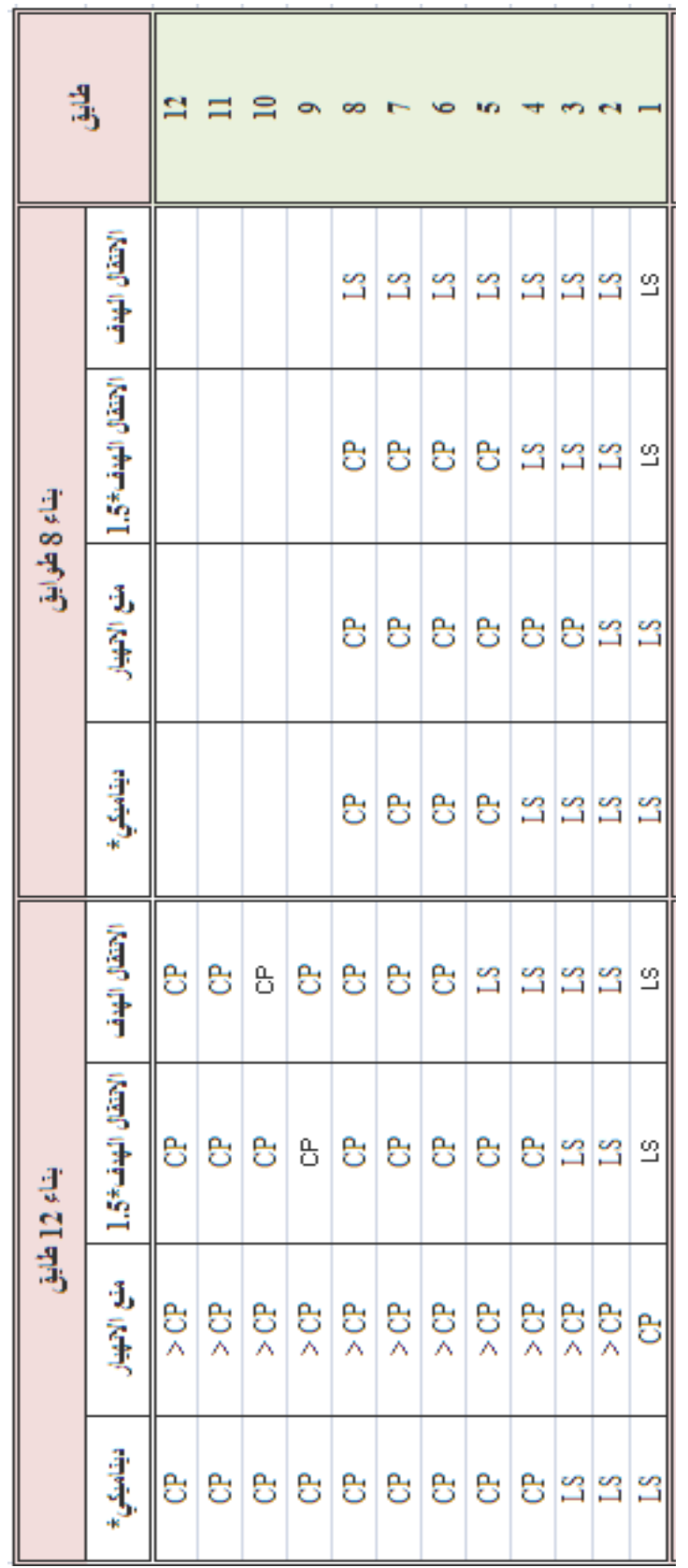




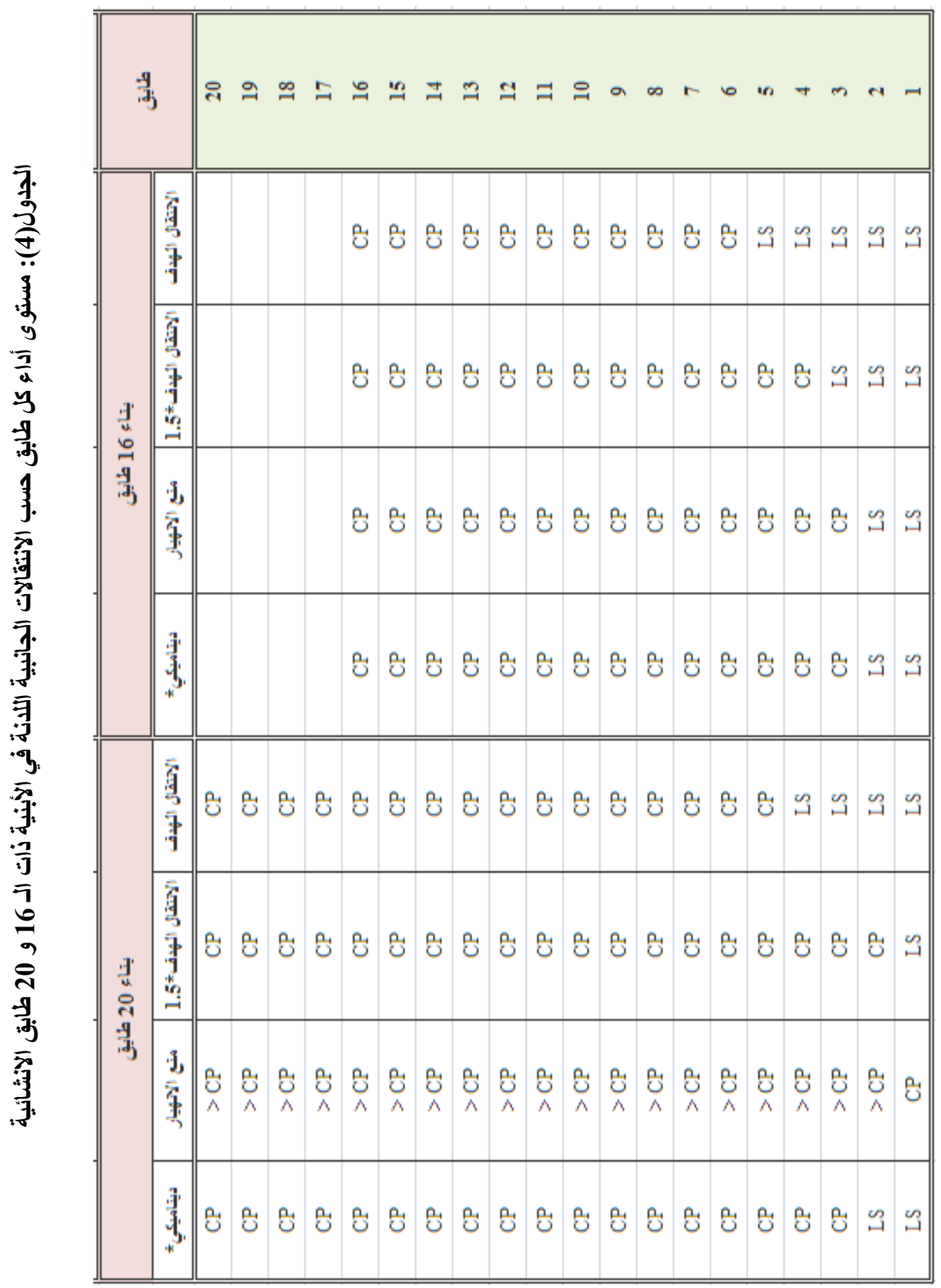


وقد كانت مستويات أداء الأبنية الدروسة حسب الانتقال النسبي الجانبي لكل دور على الثكل التالي:

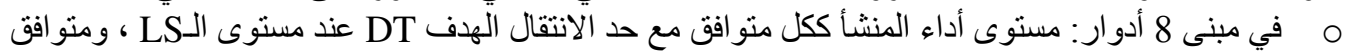

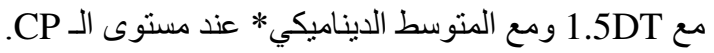

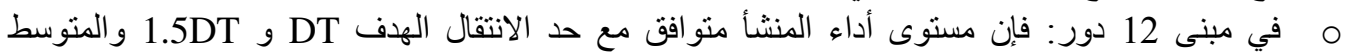
الديناميكي* عند مستوى الـCP، ومتناظر مع الانتقال اللاخطي في مرحلة الانهيار عند مستوى الـ الـ الـال .Collapse م في مبنى 16 دور: كان مستوى أداء المنشأ متناظر مع حد الانتقال الهدف DT و 1.5DT ومع الدتوسط

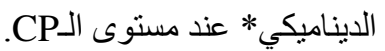

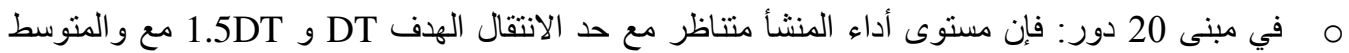

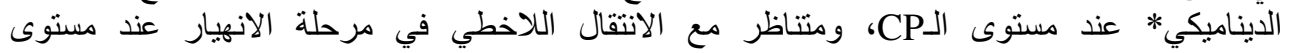
الـ Collapse. تلخص هذه النتائج في الجدول (5).

الجدول(5): مستوى أداء الأبنية المدروسة حسب الدوران اللان، الانتقالات الجانبية النسبية ومؤشر الخسارة|لانثائي

\begin{tabular}{|c|c|c|c|c|c|c|}
\hline \multicolumn{3}{|c|}{ بناء 12 طابق } & \multicolumn{3}{|c|}{ بناء 8 طوابق } & \multirow{2}{*}{ ثـ تبين سنتوى أنداء } \\
\hline هؤشر ضرن & الانحراف الجانبي & الدوبان التلان & هؤشر ضرن & الأحراف الجاتبي & الدودان التلان & \\
\hline M & $\mathrm{CP}$ & $\mathrm{CP}$ & M & LS & LS & الاتنتال الهنق \\
\hline S & $\mathrm{CP}$ & $\mathrm{CP}$ & S & $\mathrm{CP}$ & LS & الانتقل الهيف 1.5 \\
\hline S & $>\mathrm{CP}$ & $>\mathrm{CP}$ & S & $\mathrm{CP}$ & LS & تئ الانهياز \\
\hline $\mathrm{s}$ & $\mathrm{CP}$ & $\mathrm{CP}$ & $\mathrm{s}$ & $\mathrm{CP}$ & LS & التنوسط الليناسيكيخ \\
\hline S & & $>\mathrm{CP}$ & S & & $\mathrm{CP}$ & النتوسط الثيتانيكي \\
\hline \multicolumn{3}{|c|}{ بناء 20 طابق } & \multicolumn{3}{|c|}{ بناء 16 طابق } & هـ تعين مستوى أناءو \\
\hline هؤشر ضرن & الانحراف الجانبي & الدودان الثلان & هؤشر ضرن & الانحراف الجاتببي & الدونان اللان & النثشأت \\
\hline $\mathrm{s}$ & $\mathrm{CP}$ & $\mathrm{CP}$ & M & $\mathrm{CP}$ & $\mathrm{CP}$ & الاتنتال الشتف \\
\hline S & $\mathrm{CP}$ & $>\mathrm{CP}$ & S & $\mathrm{CP}$ & $>\mathrm{CP}$ & الأنقال الهن +1.5 \\
\hline S & $>\mathrm{CP}$ & $>\mathrm{CP}$ & & & & ني الانهياز \\
\hline $\mathrm{s}$ & $\mathrm{CP}$ & $>\mathrm{CP}$ & $\mathrm{s}$ & $\mathrm{CP}$ & $>\mathrm{CP}$ & التنوسط الثينانيكيخ \\
\hline $\mathrm{S}$ & & $>\mathrm{CP}$ & $\mathrm{s}$ & & $\mathrm{CP}$ & اليتوسط الدينانيكي \\
\hline
\end{tabular}

تظهر مقارنة النتائج الواردة في الجدول (3) مع النتائج في الجداول (3) و (4) أن الحصول على مستوى الأداء

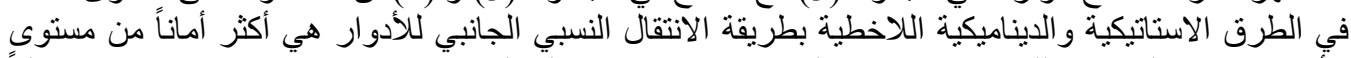

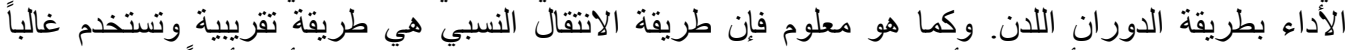
للحصول على مستوى أداء المنثأ ككل و لا يمكن مقارنتها مع طريقة الدور الان الدان اللان كونها أكثر أماناً.

4-5 المرحلة الرابعة ـ مؤشر ضرر العناصر والمنشأ بأكمله

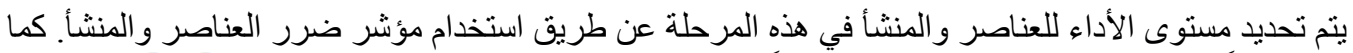

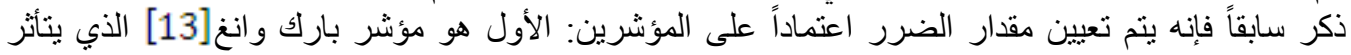

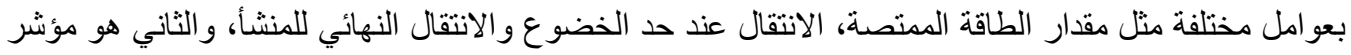


قبار المبني على تغيرات القساوة. للحصول على مقدار الضرر حسب علاقة القساوة فيجب تحويل منحني الاسنطاعة (قوة - انتقال) إلى منحني مؤلف الف الفئ من خطين.

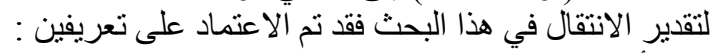

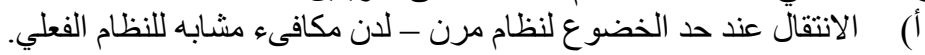

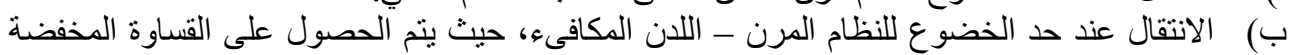

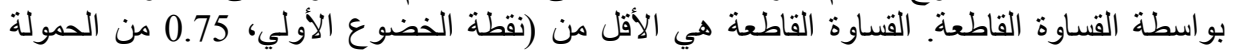

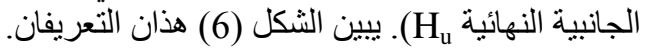

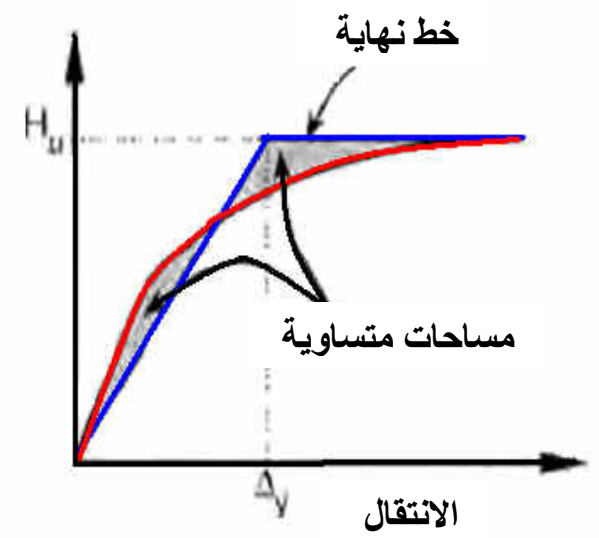

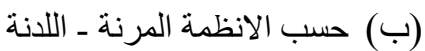
بطريقة امتصاص الطاقة المعادلة

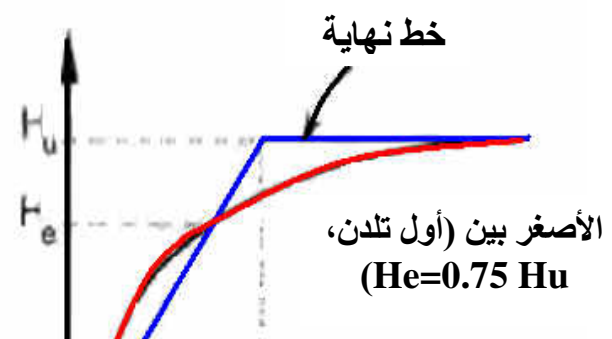

(He=0.75 Hu الانتقال (أ) حسب الأنظمة المرنة_ اللانة بطريقة القساوة المخفضة المعادلة

الشكل(6): طريقة تمثيل (تحويل) منحنيات السعة أو الاستجابة الكلية للمبنى (بشكل خطين عوضاً عن

[41] المنحني)

تم الاستفادة من التعريف (أ) بسبب استخدامه في كود التأهيل كما تم الاستفادة من التعريف (ب) لأنه يعطي

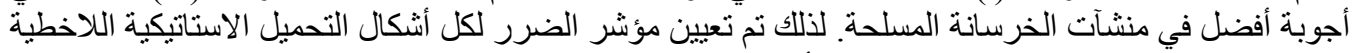

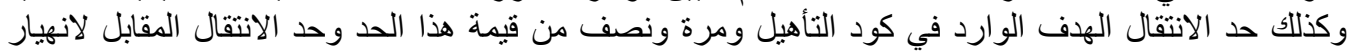

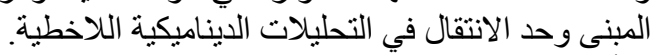

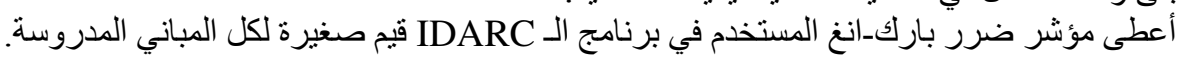

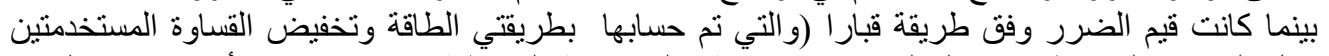

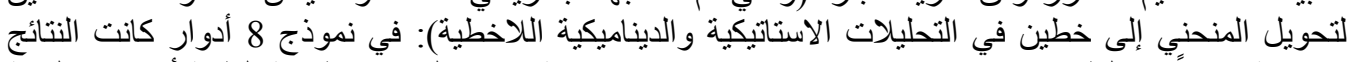

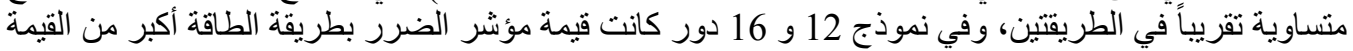

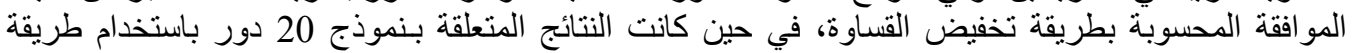

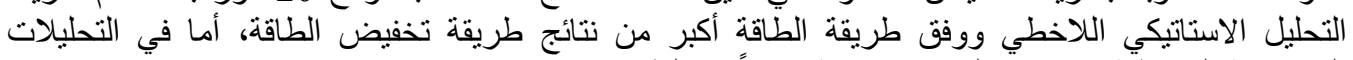

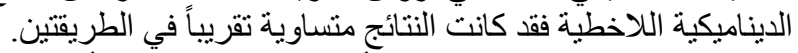

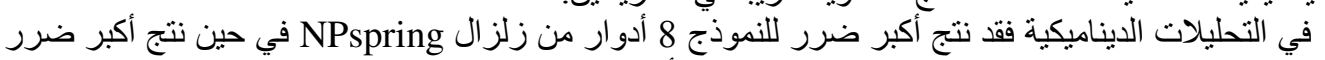

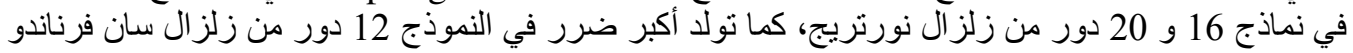

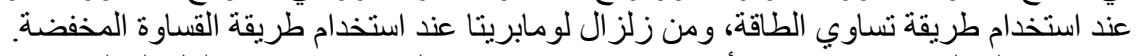

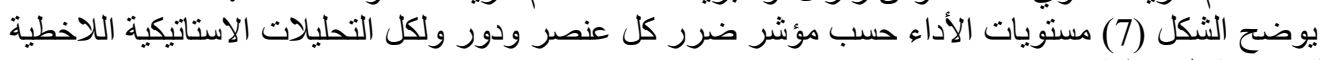



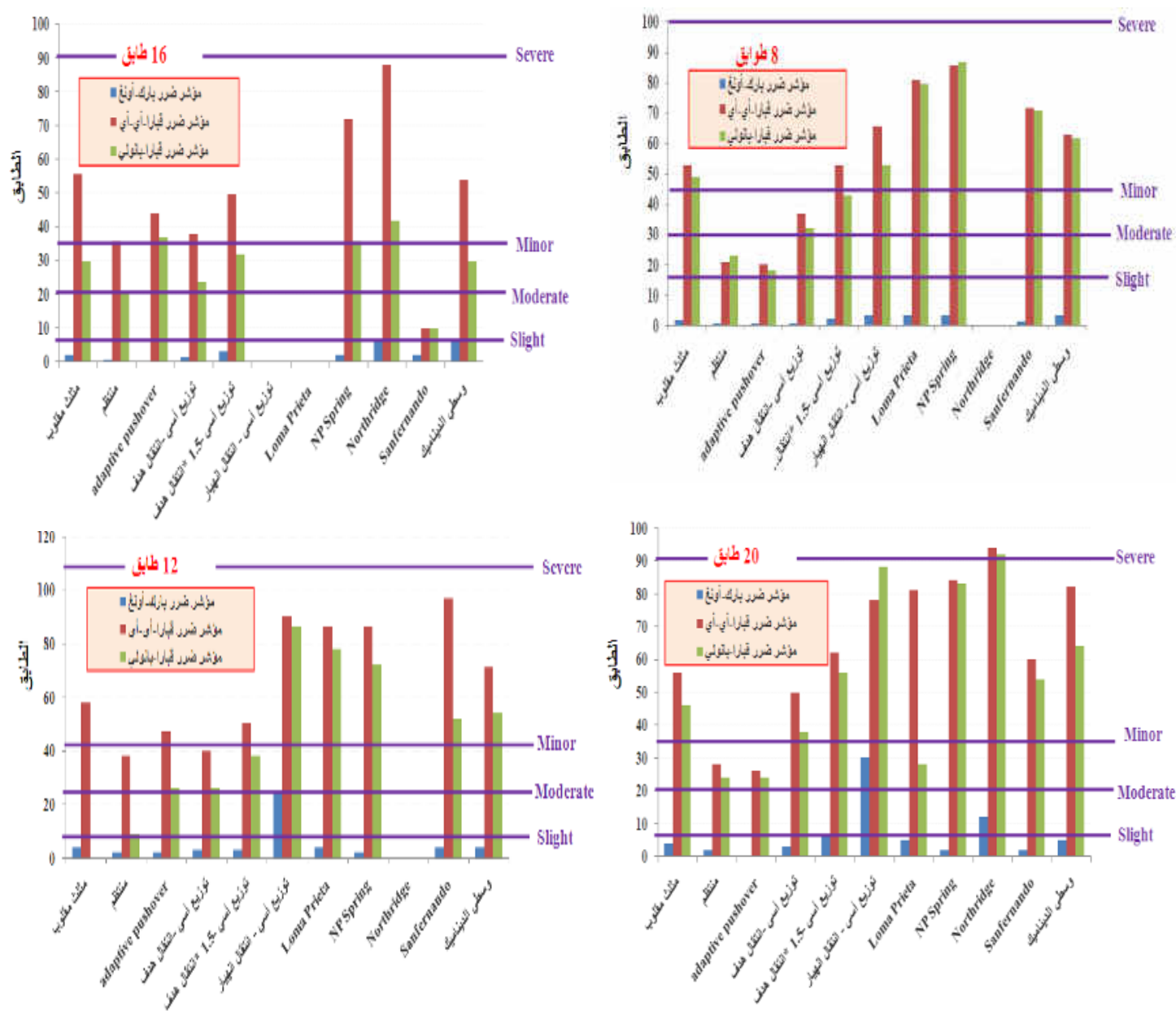

الشكل(7): مقدار الخسارة الحاصلة للمنشآت المدروسة حسب مؤشر خسارة قبار او بارك-أونغ

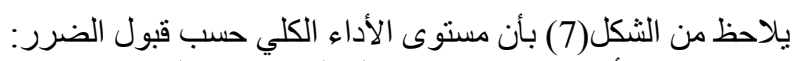

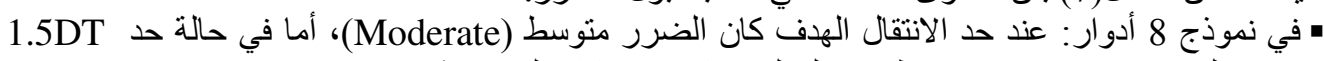

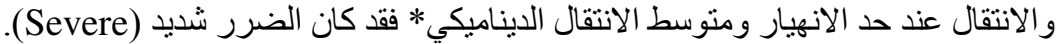

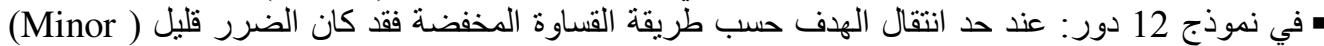

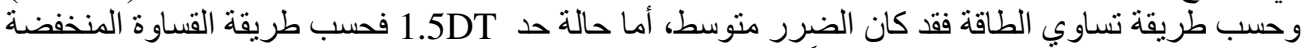

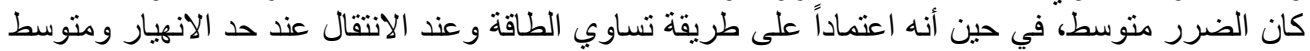
الانتقال الديناميكي* فقد كان الضرر شديدة (

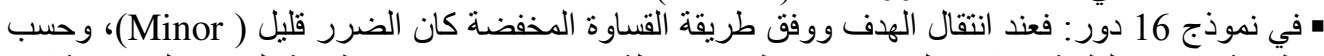
طريقة تساوي الطاقة فقد كان الضرر منوسط، وفي حالة حد

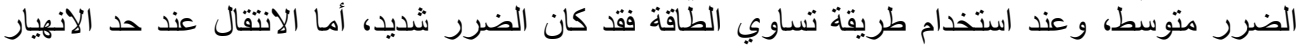

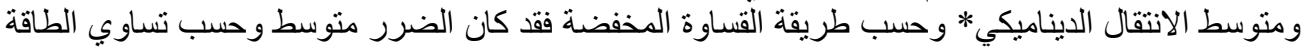
كان الضرر شديد( (Severe). • في نموذج 20 دور : في انتقال الهدف حسب طريقة القساوة المخفضة كان الضرر منوسط، وحسب طريقة تساوي الطاقة فقد كان الضرر شديد، وفي حالة حد

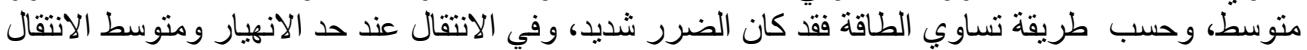
الديناميكي * كان الضرر شنديد. 


\section{6 - 6تنجة}

تم التوصل إلى النتائج التالية في النظم الإنشائية المختلطة والتي يمكن تعميمها على جميع أنواع النظم

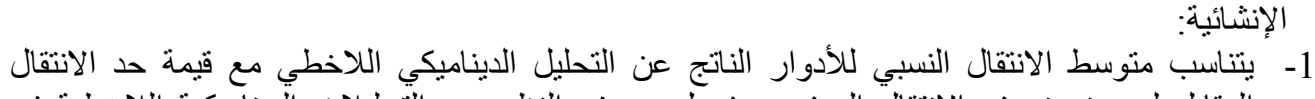

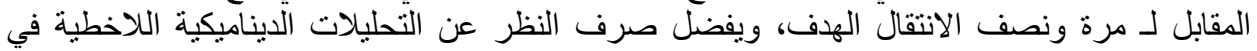
الحالات التي يكون من المعقد تفسير نتائجها واستخدام قيم الانتقالات النسبية الناتجة من التحليلات الاستاتيكية التلاخطية بدلاً منها.

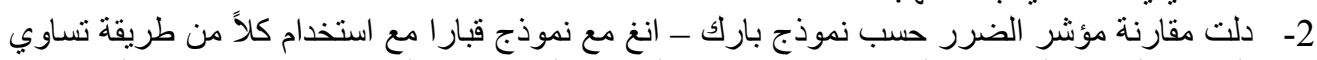

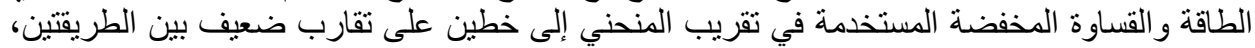

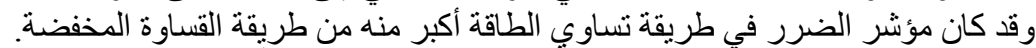

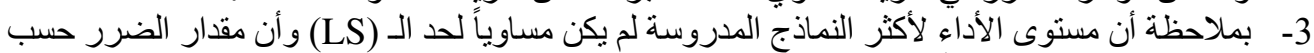

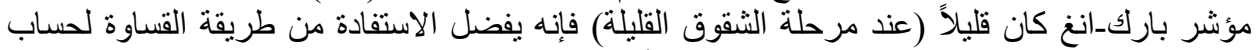

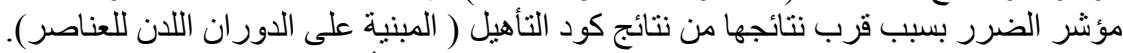

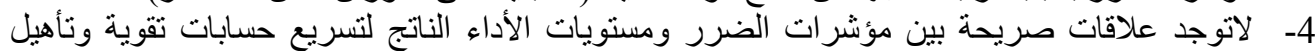

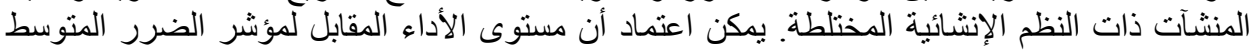

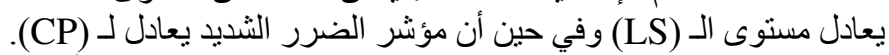

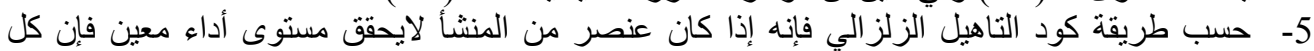

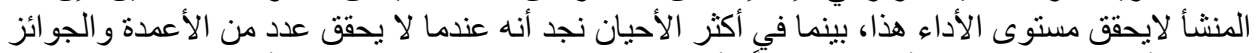

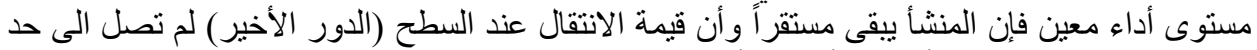

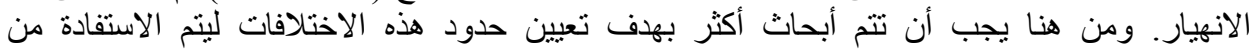
الاستطاعة ومستوى الأداء الحقيقيين في تصميم تقوية وتأهيل هذا النوع من الأبنية .

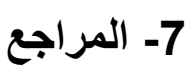

1. Abrams, D.P. (1980). "Experimental Study of Reinforced Concrete Frame-Wall Structures Subjected to Strong Earthquake Motions", Proceedings of the 7th World Conference on Earthquake Engineering, 191-198.

2. Kunnath, S.K. et al (1990). "Analytical Modeling of Inelastic Seismic Response of RC Structures", Jour. Struc. Engg. 116(4).

3. Liao, Wen-I. et al (2004). "Experimental Studies of High Seismic Performance Shear Walls", Proc. of 13th World Conf. on Earthquake Engineering, Vancouver, B.C. Canada, Paper No. 501.

4. Maheshw, A. and Santhakumar, A.R. (2004). "Capacity Design for Tall Buildings with Mixed System", 13th World Conference on Earthquake Engineering, Vancouver, B.C., Canada, Paper No. 2367.

5. Hong-Nan, L. and Bing, L. (2004). "Experimental Study on Seismic Restoring Performance of Reinforced Concrete Shear Walls", 13th World Conference on Earthquake Engineering,Vancouver, B.C., Canada , Paper No. 1559.

6. Ranai, R.J.L. and Zekioglu, A. (2004). "Pushover Analysis of 19 Story Concrete Shear Wall Building," 13th World Conference on Earthquake Engineering, Vancouver, B.C., Canada, Paper No.133.

7. Applied Technology Council (1996). "Seismic Evaluation and Retrofit of Concrete Buildings", Report ATC 40.

8. Federal Emergency Management Agency (1997). "Guidelines for the Seismic Rehabilitation of Buildings", FEMA 273. 
9. Federal Emergency Management Agency (1997). "Commentary on the Guidelines for the Seismic Rehabilitation of Buildings", FEMA 274.

10. Federal Emergency Management Agency (2000). "Prestandard and Vommentary for the Seismic Rehabilitation of Buildings", FEMA 356.

11. Federal Emergency Management Agency (2000). "Evaluation of Earthquake Damaged Concrete and Masonry Wall Buildings, Basic Procedures Manual", FEMA 306.

12. Federal Emergency Management Agency (2000). "Evaluation of Earthquake Damaged Concrete and Masonry Wall Buildings, Technical Resources", FEMA 307.

13. Park, Y.J. and Ang, A.H-S. (1985). "Mechanistic Seismic Damage Model for Reinforced Concrete, Journal of Structural Engineering, ASCE, 11(ST4), 722-739.

14. Park, Y.J., Ang, A.H-S., and Wen, Y.K. (1985). "Seismic Damage Analysis of Reinforced Concrete Buildings", Journal of Structural Engineering, ASCE, 11(ST4), 740-757.

15. Ghobarah, A., Abou-Elfath, H., and Biddah, A. (1999). "Response-Based Damage Assessment of Structures", Earthquake Engineering Structural Dynamics, 28, 79104.

16. Banon, H. et al (1981). "Seismic Damage in Reinforced Concrete Frames", Journal of Structural Engineering, ASCE, 107(ST9), 1713-1729.

17. Sordo, E., Teran, A., Geurrero, J.J., and Hglesias, J. (1989). "Ductility and Resistance Requirements Imposed on a Concrete Building", Earthquake Spectra, 5(1), 41-50.

18. Penzien, J. (1993). "Seismic Design Criteria for Transportation Structures", Structural Engineering in Natural Hazard Mitigation, Proc. ASCE Structures Congress, Irvine, CA, 1, 4-36.

19. Toussi, S. and Yao, J.T.P. (1982). "Hysteresis Identification of Existing Structures, Journal Engg. Mech. ASCE, 109(5), 1189-1203.

20. Stephens, J.E. and Yao, J.T.P. (1987). "Damage Assessment Using Response Measurements", Journal of Structural Engineering, ASCE, 113(4), 787-801.

21. Roufaeil, M.S.L. and Meyer, C. (1987a). "Analytical Modeling of Hysteretic Behavior of RC Frames", Journal of Structural Engineering, ASCE, 113(3), 429444.

22. Roufaeil, M.S.L. and Meyer, C. (1987b). "Reliability of Concrete Frames Damaged by Earthquakes", Journal of Structural Engineering, ASCE, 113(3), 445-457.

23. Wang, M.L. and Shah, S.P. (1987). "Reinforced Concrete Hysteresis Model Based in the Damage Concept", Earthquake Engineering and Structural Dynamics, 15(8), 993-1003.

24. Wang, M.L. and Wang, J. (1992). "Nonlinear Dynamic Analysis of Reinforced Concrete Shear Wall Structures", Soil Dynamics and Earthquake Engineering, 11(5), 255-268.

25. Jeong, G.D. and Iwan, W.D. (1988). "Effect of Earthquake Duration on the Damage of Structures", Earthquake Engineering and Structural Dynamics, 16(8), 1201-1211.

26. Chung, Y.S., Meyer, C., and Shinozuka, M. (1989a). "Modeling on Concrete Damage", Struc. Jour. American Concrete Institute, 86(3), 259-271. 
27. Chung, Y.S., Meyer, C., and Shinozuka, M. (1989b). “Automated DamageControlled Design of RC Buildings", Proc. 5th Int. Conf. on Structural Safety and Reliability (ICOSSAR 89), San Francisco, CA, 1, 383-390.

28. Chung, Y.S., Meyer, C., and Shinozuka, M. (1990). "Automated Seismic Design of Reinforced Concrete Building Frames", Structural Journal American Concrete Institute, 87(3), 326-340.

29. Gosain, N.K., Brown, R.H., and Jirsa, J.O. (1977). "Shear Requirements for Load Reversals on RC Members", Journal of Structural Engineering, ASCE, 103(7), 1461-1476.

30. Darwin, D. and Nmai, C.K. (1986). "Energy Dissipation in RC Beams under Cyclic Load", Journal of Structural Engineering, ASCE, 112(8), 1829-1846.

31. Elms, D., Paulay, T., and Ogawa, S. (1989). "Code-Implied Structural Safety for Earthquake Loading”, Proc. 5th Int. Conf. on Structural Safety and Reliability (ICOSSAR 89), San-Francisco, CA, 3, 2003-2010.

32. Kratzig, W.B., Meyer, I.F., and Meskouris, K. (1989). "Damage Evolution in Reinforced Concrete Members under Cyclic Loading", Proc. 5th Int. Conf. on Structural Safety and Reliability (ICOSSAR 89), San Francisco, CA, 2, 759-802.

33. IDARC 2D Version 4.0 (1996). "A Program for the Inelastic Damage Analysis of RC Buildings", Technical Report NCEER-96-0010.

34. Stone, W.C. and Taylor, A.W. (1993). "Seismic Performance of Circular Bridge Columns Designed in Accordance with AASHTO/ CALTRANS Standards, NIST Building Science Series 170, National Institute of Standards and Technology, Gaithersburg, MD.

35. Otani, S. and Sozen, M.A. (1972). "Behavior of Multi-Storey Reinforced Concrete Frames During Earthquakes", Structural Research Series No. 392, Civil Engineering Studies, University of Illinois, Urbana, IL.

36. Paulay, T. and Priestley, M.I.N. (1992). "Seismic Design of Reinforced Concrete and Masonry Buildings", John Willy \& Sons, New York. 